\title{
Chemical characterization of the inorganic fraction of aerosols and mechanisms of the neutralization of atmospheric acidity in Athens, Greece
}

\author{
E. T. Karageorgos and S. Rapsomanikis \\ Laboratory of Atmospheric Pollution and Pollution Control Engineering, Faculty of Engineering, Department of \\ Environmental Engineering, Democritus University of Thrace, P.O. Box 447, 67100 Xanthi, Greece
}

Received: 31 July 2006 - Published in Atmos. Chem. Phys. Discuss.: 1 December 2006

Revised: 7 May 2007 - Accepted: 26 May 2007 - Published: 12 June 2007

\begin{abstract}
The $\mathrm{PM}_{10}$ mass concentration levels and inorganic chemical composition were determined on 12-h resolution sampling during August 2003 and March 2004, in the centre of Athens, Greece. The August 2003 campaign mean $\mathrm{PM}_{10}$ mass concentration, obtained by Beta Attenuation at $5 \mathrm{~m}$ above ground in Athinas Street, was $56 \mu \mathrm{g} \mathrm{m}^{-3}$ while the corresponding value for March 2004 was $92 \mu \mathrm{g} \mathrm{m}^{-3}$. In both campaigns the E.U. imposed daily limit of $50 \mu \mathrm{g} \mathrm{m}^{-3}$ was exceeded on several days. During the March campaign, in Athinas Street, additionally obtained DSFU-PM 10 $\left(\mathrm{PM}_{10-2.5}+\mathrm{PM}_{2.5}\right)$ gravimetric mass concentrations (mean: $121 \mu \mathrm{g} \mathrm{m}^{-3}$ ) in the "breathing zone", at $1.5 \mathrm{~m}$ above ground were significantly higher compared to the respective mean $\mathrm{PM}_{10}$ mass concentrations obtained by the same method at $25 \mathrm{~m}$ above ground, in a second site (AEDA; mean: $86 \mu \mathrm{g} \mathrm{m}^{-3}$ ) also in the centre of the city. The above findings suggest that, for a realistic estimation of the exposure of citizens to particulate matter, $\mathrm{PM}_{10}$ sampling in the "breathing zone" (1.5-3 m above ground) is necessary. Such data are presented for the first time for the centre of Athens. In both campaigns, calcium was found to be the predominant component of the coarse fraction while crust-related aluminosilicates and iron were the other major components. The above elements constitute the most important components of the fine fraction, together with the predominant sulphur. All toxic metals were found in concentrations below the established air quality limits, and most of them in lower concentrations compared to older studies. Lead in particular, appeared mostly in the fine fraction and in very low concentrations compared to studies dating more than a decade back. The predominant ions of the coarse fraction have been found to be $\mathrm{Ca}^{2+}, \mathrm{NO}_{3}{ }^{-}, \mathrm{Na}^{+}$and $\mathrm{Cl}^{-}$, while $\mathrm{SO}_{4}{ }^{2-}, \mathrm{Ca}^{2+}$ and $\mathrm{NH}_{4}{ }^{+}$were the major ionic components of the fine fraction. In the fine particles, a low molar ratio of $\mathrm{NH}_{4}{ }^{+} / \mathrm{SO}_{4}{ }^{2-}$ in-
\end{abstract}

Correspondence to: E. T. Karageorgos

(vkarageo@env.duth.gr) dicated an ammonium-poor ambient air, and together with inter-ionic correlations suggested that atmospheric ammonia is the major neutralizing agent of sulfate, while being insufficient to neutralize it to full extend. The formation of $\mathrm{NH}_{4} \mathrm{NO}_{3}$ is therefore not favored and additional contribution to the neutralization of acidity has been shown to be provided by $\mathrm{Ca}^{2+}$ and $\mathrm{Mg}^{2+}$. In the coarse particle fraction, the predominantly abundant $\mathrm{Ca}^{2+}$ has been found to correlate well with $\mathrm{NO}_{3}^{-}$and $\mathrm{SO}_{4}{ }^{2-}$, indicating its role as important neutralizing agent in this particle size range. The proximity of the location under study to the sea explains the important concentrations of salts with marine origin like $\mathrm{NaCl}$ and $\mathrm{MgCl}_{2}$ that were found in the coarse fraction, while chloride depletion in the gaseous phase was found to be limited to the fine particulate fraction. Total analyzed inorganic mass (elemental+ionic) was found to be ranging between approximately $25-33 \%$ of the total coarse particle mass and $35-42 \%$ of the total fine particle mass.

\section{Introduction}

The concentration levels, chemical composition and physical properties of urban aerosols depend on the nature of their emission sources, on their dispersion and on their subsequent chemical reactions in the atmosphere.

Throughout the last decades, a number of epidemiological studies have clearly demonstrated that aerosol pollution constitutes a potential hazard to citizens (Dockery and Pope, 1994; Touloumi et al., 1994; Katsouyanni et al., 1997; Katsouyanni et al., 2001; Pope III et al., 2002; Peng et al., 2005). In relation to this, special attention has been drawn on atmospheric pollution from particles in the "inhalable" size range, $\mathrm{PM}_{10}$ and their finer, "respirable" fraction, $\mathrm{PM}_{2.5}$. The issue is of greater importance when it refers to largely populated urban environments, where often, high particulate pollution phenomena are observed. An integral part of improving air

Published by Copernicus Publications on behalf of the European Geosciences Union. 
quality in the E.U. is the identification of the chemical composition and the principal sources of particulate pollutants, so as to permit the most effective, source-targeting, control strategies.

Athens, Greece, has an estimated population of 4 million inhabitants. The city is built in a $400 \mathrm{~km}^{2}$ shallow valley, surrounded by moderate height hills to the north, west and east and the Saronikos Gulf to the south and south west. The hills surrounding the area are considered largely responsible for the poor dispersion conditions often observed in the city. Traffic is considered as a major source of anthropogenic atmospheric pollution in the area. The total number of vehicles circulating over the Greater Athens Area (GAA) exceeds 2 million. Of these, approximately 800000 Light Duty Vehicles (LDV's) are non-catalytic, while ca. 100000 Heavy Duty Vehicles (HDV's) and taxies are diesel-powered (Symmeonidis, 2002). Other important causes of anthropogenic atmospheric pollution include industrial activity, as some of the country's major heavy industries are located right outside the valley, West and North West of the city, and domestic heating during the colder season.

Particulate matter pollution in Athens and the GAA has been the subject of several studies carried out during the past 20 years. Among the studied parameters is the fractionation and spatio-temporal variation of PM mass concentrations in GAA (Chaloulakou et al., 2003; Grivas et al., 2004), the concentrations of organic, elemental and inorganic carbon (Sillanpää and al., 2005) and some toxic metals in the PM (Scheff and Valiozis, 1990; Koliadima et al., 1998; Thomaidis et al., 2003; Manalis et al., 2005; Sillanpää et al., 2006) and of some ionic species in $\mathrm{PM}_{2.5}$ (Scheff and Valiozis, 1990; Siskos et al., 2001; Sillanpää et al., 2006) and in TSP (Eleftheriadis et al., 1998). In addition to the above, the monitoring network of the Ministry of Environment, Planning and Public Works, has been modernized and since 2000 the mass concentration of $\mathrm{PM}_{10}$ as well as the concentrations of a series of gaseous pollutants, are determined on a daily basis, in a series of sites throughout the city.

While the aforementioned studies have aided in an understanding of the aerosol pollution in Athens, to a significant level, thorough knowledge of the chemical composition of the PM is still not existent. In addition to this, the governmental monitoring network and the aforementioned studies, in their entirety, have entailed aerosol sampling carried out at heights ranging from 7 to $25 \mathrm{~m}$ above ground. The present study aims to determine the concentrations and a more detailed inorganic chemical composition of the PM, not only at the aforementioned height levels, but also near the ground, in the "breathing zone" (Commission, 1999/30/EC), as this is indicative of the levels of pollution to which thousands of citizens, moving in the centre of the city, are exposed on a daily basis.

The detailed objectives of the present study are, firstly, the determination of the inorganic chemical composition "fingerprint" of fine and coarse particles in the centre of Athens.
Secondly, the comparison between the mass concentrations and chemical composition of coarse and fine particles collected near the ground and particles collected on a building roof, in the centre of the city. Thirdly, the comparison of total particulate matter, as well as metals and ions concentrations with the ones reported in previous studies for the city and in studies for other major urban centers of the world. Fourthly, the determination of the seasonal variation of mass concentration and chemical composition of the PM in the centre of Athens.

\section{Experimental}

\subsection{Sampling}

Aerosol sampling took place during two periods, the first between 7 and 27 August 2003 and the second between 8 and 28 March 2004. In both periods, sampling was carried out at a site located behind the Athens City Hall, in Athinas Street $\left(\approx 12000\right.$ vehicles/day) $\left(38^{\circ} 02^{\prime} \mathrm{N}, 23^{\circ} 44^{\prime} \mathrm{E}\right)$. Athinas St. normally has a constant traffic load, largely due to the nearby Athens central market. At the time of the sampling periods, both in August 2003 and especially in March 2004, a series of construction activities were taking place at several points across Athinas St., due to the preparations for the Olympic Games of 2004. This resulted in a significant reduction of the traffic load. The sampling equipment on the first site consisted of a pair of Dichotomous Stack Filter Units (DSFU) (Luhana L. et al., 2001) for the collection of filter samples and a Particle Analyzer measuring mass concentration by use of Beta-Attenuation (FH62I-R, ESM-Thermo Andersen; hereafter referred to as BA), also equipped with a reflectometer for the determination of Black Carbon. Additional sampling during March took place on the roof of a governmental building (AEDA) also in downtown Athens. Blocks of 4 to 6-storey buildings dominate the location. The distance between the two sampling sites is approximately $700 \mathrm{~m}$ and their exact locations are shown in Fig. 1. Sampling at AEDA took place by means of 2 DSFUs. The DSFU samplers' inlets in Athinas St. were set at a height of $1.5 \mathrm{~m}$ above ground level. The Beta Attenuation Particle Analyzer inlet was set at a height of approximately $5 \mathrm{~m}$ from the ground level. In AEDA, the DSFU sampling took place at a height of approximately $25 \mathrm{~m}$ from the street level.

For the flow-control and measurement, four new 810C Mass-TraksTM (Full Model No.: 810C-CE-DR-2; SIERRA Instruments, Inc., Los Angeles, USA) were used. The devices have an accuracy of $\pm 1 \%$ and a maximum systematic reading error of $\pm 0.2 \%$. All four devices had been calibrated with air (calibration Method: Sierra Cal-Bench; Sierra Instruments) based on the US National Institute of Standards and Technology method). At the flow-rate of $6.0 \mathrm{~L} / \mathrm{min}$, that was employed during our campaigns, the $50 \%$ efficiency cut-off point is $10.1 \pm 0.5 \mu \mathrm{m}$ for the DSFU pre-impactor, 

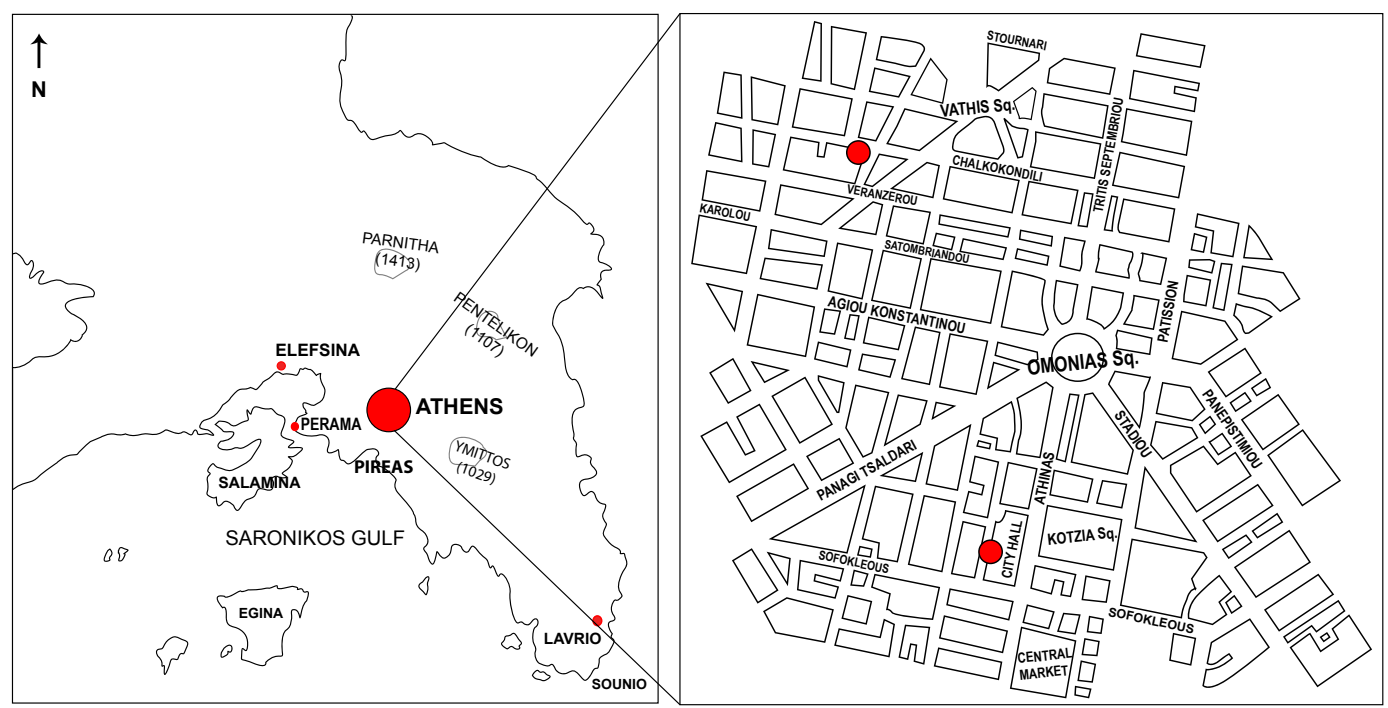

Fig. 1. Map of Athens showing the sampling sites.

and $2.8 \pm 0.2 \mu \mathrm{m}$ for the upper $12 \mu \mathrm{m}$ filter (Luhana L. et al., 2001). As a result of this, particles collected were divided into two fractions; coarse, with an aerodynamic diameter between $2.8 \mu \mathrm{m}$ and $10 \mu \mathrm{m}$ (hereafter referred to as CPM), and fine, with an aerodynamic diameter smaller than $2.8 \mu \mathrm{m}$ (hereafter referred to as FPM). Particles with an aerodynamic diameter greater than $10 \mu \mathrm{m}$, were trapped on a lightly greased pre-impactor surface, in the front part of the stack. The flowrate was essentially constant, varying within approx. $\pm 2 \%$, throughout the 12 -h measurement sessions. For an estimation of the precision of the flow control/measurement in the pairs of DSFUs, the volumes of air sampled with each sampler were regressed to one another and the relationship between them was found to be $y=0.988$ $x-0.018\left(R^{2}=0.97\right)$. The relationship between the respective sampled masses of particulate matter, with the two samplers was found to be described by the equation: $y=0.965 x+1.03$ $\left(R^{2}=0.96\right)$. Polycarbonate Membrane filters (Whatman) with a pore-diameter of $12 \mu \mathrm{m}$ and $0.4 \mu \mathrm{m}$ were used for the collection of CPM and FPM respectively. In order to avoid bounce-off phenomena, 12- $\mu \mathrm{m}$ filters were coated with a thin layer of Apiezon-L grease (Apiezon products, London, UK) (Harrison et al., 1996). All filters were weighed on a micro balance with an accuracy of $10 \mu \mathrm{g}$ (Scaltec SBC22), before and after sampling, thus allowing the gravimetric determination of CPM and FPM mass concentrations. Prior weighing, filters were kept under stable environmental conditions (RH: $\sim 30 \%, T=18^{\circ} \mathrm{C}$ ) in vacuum desiccators for 24 hours. Every sampling day was divided into two periods; a morning period from 07:00 to 19:00 and an evening period from 19:00 to $07: 00$. Field blanks were collected at the beginning and the end of the sampling periods by placement of filters in the DSFU samplers for a complete sampling session with sampling pumps shut down.
Some FPM filters broke during the campaign, due to the vacuum behind them when they were overloaded. Moreover, in March 2004, during two weekends and a national holiday (25 March) access to the AEDA site, in the evening, was restricted due to the preparation of security measures for the then forthcoming Olympic Games. In those cases, as the change of filters in the evening was not possible, the sampling sessions in AEDA were necessarily 24-h long, which again resulted in the breaking of some FPM filters. The broken FPM filters have been excluded from the subsequent calculations and statistical analysis.

Meteorological data including wind speed and direction, relative humidity, temperature and solar radiation, as well as major urban gas-pollutants concentrations $\left(\mathrm{O}_{3}, \mathrm{NO}+\mathrm{NO}_{2}\right.$, $\mathrm{CO}, \mathrm{SO}_{2}$ ), were obtained from the Hellenic Ministry of Environment. The meteorological stations were located in central parts of Athens, close to Athinas St. and AEDA.

\subsection{Extraction and analysis}

The use of two DSFUs in each of the sites, made it possible to obtain pairs of virtually equally loaded ("twin") filters for every sampling session. One of the "twin" filters was analyzed by Proton Induced X-Ray Emission (PIXE) for identification and quantification of 24 metal species with an atomic number $>12$, in the Department of Atmospheric Environment (NERI, Denmark) in accordance to the ISO 17025 standard (DANAK, Danish Accreditation no. 411, laboratory accredited for PIXE analysis). The second of the "twin" filters was analyzed for quantification of major anions $\left(\mathrm{NO}_{3}^{-}, \mathrm{PO}_{4}^{3-}\right.$, $\left.\mathrm{SO}_{4}^{2-}, \mathrm{Cl}^{-}\right)$and cations $\left(\mathrm{Na}^{+}, \mathrm{NH}_{4}^{+}, \mathrm{K}^{+}, \mathrm{Mg}^{2+}, \mathrm{Ca}^{2+}\right)$ in the water soluble fraction, by Ion Chromatography (IC), in the Laboratory of Atmospheric Pollution and Pollution Control Engineering (Department of Environmental Engineering, 
Table 1. PIXE detection limits for ambient air samples on membrane filters of mixed cellulose esters (a.d.: area of deposit=13 $\mathrm{cm}^{2}$ ).

\begin{tabular}{cccccccc}
\hline & $\mu$ g/a.d. & & $\mu$ g/a.d. & & $\mu$ g/a.d. & & $\mu$ g/a.d. \\
\hline $\mathrm{Al}$ & 1.9 & $\mathrm{Ti}$ & 0.02 & $\mathrm{Zn}$ & 0.05 & $\mathrm{Zr}$ & 0.005 \\
$\mathrm{Si}$ & 2,3 & $\mathrm{~V}$ & 0.017 & $\mathrm{Ga}$ & 0.007 & $\mathrm{Mo}$ & 0.006 \\
$\mathrm{P}$ & 1.8 & $\mathrm{Cr}$ & 0.017 & $\mathrm{As}$ & 0.006 & $\mathrm{Cd}$ & 0.03 \\
$\mathrm{~S}$ & 0.8 & $\mathrm{Mn}$ & 0.012 & $\mathrm{Se}$ & 0.002 & $\mathrm{Sn}$ & 0.04 \\
$\mathrm{Cl}$ & 0.6 & $\mathrm{Fe}$ & 0.14 & $\mathrm{Br}$ & 0.007 & $\mathrm{Sb}$ & 0.06 \\
$\mathrm{~K}$ & 0.3 & $\mathrm{Ni}$ & 0.012 & $\mathrm{Rb}$ & 0.004 & $\mathrm{Ba}$ & 0.2 \\
$\mathrm{Ca}$ & 0.6 & $\mathrm{Cu}$ & 0.02 & $\mathrm{Sr}$ & 0.004 & $\mathrm{~Pb}$ & 0.014 \\
\hline
\end{tabular}

Democritus University of Thrace). In August 2003 ionic concentrations were not measured.

\subsubsection{Elemental analysis}

A calculation of the absolute standard deviation for each PIXE-analysed element is incorporated in the fit of the $\mathrm{x}$ ray spectrum. The uncertainties and detection limits depend on the number of x-ray counts, the thickness of the filter material (due to the continuous spectral background), its purity and the matrix (interferences from neighbour spectral peaks). Table 1 shows detection limits for typical ambient air samples on mixed cellulose ester filters, using a proton radiation time of approximately $20 \mathrm{~min}$ per sample. The detection limit value is either the average standard deviation of the values in a series of blanks, or the standard deviation of the blank values, depending on which is the largest. PIXE detection limits are estimated as mass per area. The uncertainty of the integrated proton charge and fluctuations of the detector solid angle give rise to an additional standard deviation of $5 \%$, which is geometrically added to the spectral uncertainty. The calibration is permanent and is regularly controlled. The calibration uncertainty for all elements is $7 \%$ on average and, as a result of the above, far from the detection limit the average accuracy is on average $9 \%$ for all elements (the geometric sum of 5\% and 7\%). For semi-thick samples, which need correction for x-ray self-absorption, a non-stratified (homogeneous) mixture of elements is assumed in the mass-perarea calculation. An extra uncertainty is included due to the possible stratification of the sample. Individual uncertainties have been calculated for every compound and every sample of the campaign. The PIXE analysis as a minimum includes the elements $\mathrm{Al}, \mathrm{Si}, \mathrm{S}, \mathrm{Cl}, \mathrm{K}, \mathrm{Ca}, \mathrm{Ti}, \mathrm{V}, \mathrm{Cr}, \mathrm{Mn}, \mathrm{Fe}, \mathrm{Ni}, \mathrm{Cu}$, $\mathrm{Zn}, \mathrm{Ga}, \mathrm{As}, \mathrm{Se}, \mathrm{Br}, \mathrm{Rb}, \mathrm{Sr}, \mathrm{Zr}, \mathrm{Mo}, \mathrm{Cd}, \mathrm{Sn}, \mathrm{Sb}, \mathrm{Ba}, \mathrm{Pb}$, as well as others (e.g. P, I, W, Th) in the case they are present in significant amounts. The quantification of $\mathrm{Cl}$ is not considered very reliable, because a percentage of $\mathrm{HCl}$ is lost, during the PIXE analysis. The maximum possible loss of $\mathrm{Cl}$ is the equivalent quantity of sampled acidic compounds (organic acids, $\mathrm{H}_{2} \mathrm{SO}_{4}, \mathrm{HSO}_{4}^{-}$and $\mathrm{NH}_{4}^{+}$).

\subsubsection{Ions analysis}

A DX-500 Chromatograph (Dionex Corp., Sunnyvale, CA, USA) with an AS-9HC column for anions and a CS12 column for cations were used for the ions analysis. An ASRSUltra II and a CSRS-Ultra suppressors, working in AutoRecycle mode, were employed for the eluent suppression. All solid filter samples to be analyzed by IC were kept in a closet, under controlled room temperature $\left(18^{\circ} \mathrm{C}\right)$ until the day of extraction. Extraction in Deionized Water $\left(18.2 \mathrm{M} \Omega \mathrm{cm}^{-1}\right)$ was done by 10 -min vigorous shaking and 30-min ultrasonication and in batches of 20 filters. Three "laboratory blanks" were also prepared with every batch and analyzed together with field samples to check for potential in-laboratory contamination. All filter extracts were kept in freezer $\left(-18^{\circ} \mathrm{C}\right)$ until analysis, when they were left to equilibrate with room temperature prior to being analyzed by Ion Chromatography. The detection limit for every compound was calculated as the blank signal plus 3 standard deviations $(3 \sigma)$ of the blank (Miller and Miller, 1993). Calibration accuracy was continuously monitored by analyses of calibrationverification standards; the uncertainty was at maximum 5\%. The calibration verification standards were obtained from a commercial source (Alltech Associates, Inc., IL, USA) different to the one of the calibration standards (Dionex Corp., Sunnyvale, CA, USA). Reproducibility (precision) was calculated by 7 injections of a standard with concentrations approximately in the middle of the calibration range; the Relative Standard Deviation (RSD) was within 3\% for all compounds of interest. To check for matrix effects, the standard additions method was carried out on every 10th sample and the discrepancy between measured and calculated value was equal or lower than $3 \%$.

\section{Results and discussion}

Table 2 presents arithmetic means and standard deviations of the concentrations of metals measured in August 2003. Morning and evening values are presented separately as 
Table 2. Morning and evening concentrations $\left(\mathrm{ng} / \mathrm{m}^{3}\right.$ ) measured in Athinas St., in August 2003 (Mean \pm Standard Deviation); ${ }^{a}$ Coarse Particulate Matter $\left(\mathrm{PM}_{2.5-10}\right){ }^{\mathrm{b}}$ Fine Particulate Matter $\left(\mathrm{PM}_{2.5}\right){ }^{\mathrm{c}} \mathrm{PM}_{10}$ obtained by use of Beta Attenuation at a height of $5 \mathrm{~m}$ from ground level; gravimetrical $\mathrm{PM}_{10}$ not determined during campaign.

\begin{tabular}{|c|c|c|c|c|}
\hline & \multicolumn{4}{|c|}{ Athinas St. } \\
\hline & $\mathrm{CPM}^{\mathrm{a}}(n=35)$ & & $\mathrm{FPM}^{\mathrm{b}}(n=30)$ & \\
\hline & Morning $(n=18)$ & Evening $(n=17)$ & Morning $(n=16)$ & Evening $(n=14)$ \\
\hline $\mathrm{Al}$ & $600 \pm 176$ & $517 \pm 268$ & $351 \pm 132$ & $266 \pm 116$ \\
\hline $\mathrm{Si}$ & $1388 \pm 423$ & $1186 \pm 741$ & $544 \pm 248$ & $356 \pm 154$ \\
\hline $\mathrm{S}$ & $344 \pm 221$ & $301 \pm 155$ & $4374 \pm 1992$ & $4570 \pm 2260$ \\
\hline $\mathrm{Cl}$ & $129 \pm 96.0$ & $114 \pm 87.6$ & $43.4 \pm 24.4$ & $31.3 \pm 11.5$ \\
\hline $\mathrm{K}$ & $317 \pm 96.6$ & $292 \pm 183$ & $351 \pm 98.7$ & $339 \pm 95.0$ \\
\hline $\mathrm{Ca}$ & $5730 \pm 2321$ & $4797 \pm 4428$ & $1706 \pm 872$ & $1074 \pm 4674$ \\
\hline $\mathrm{Ti}$ & $51.4 \pm 17.1$ & $45.0 \pm 32.4$ & $22.4 \pm 10.1$ & $14.5 \pm 6.08$ \\
\hline V & $4.3 \pm 3.77$ & $6.11 \pm 3.94$ & $11.2 \pm 12.1$ & $16.3 \pm 10.4$ \\
\hline $\mathrm{Cr}$ & $4.68 \pm 2.01$ & $4.7 \pm 3.56$ & $2.43 \pm 1.29$ & $2.40 \pm 1.19$ \\
\hline Mn & $12.5 \pm 5.23$ & $11.5 \pm 7.63$ & $10.2 \pm 3.91$ & $8.01 \pm 4.3$ \\
\hline $\mathrm{Fe}$ & $690 \pm 232$ & $707 \pm 408$ & $374 \pm 138$ & $361 \pm 137$ \\
\hline $\mathrm{Ni}$ & $3.83 \pm 2.17$ & $4.05 \pm 2.59$ & $5.38 \pm 4.37$ & $5.66 \pm 3.74$ \\
\hline $\mathrm{Cu}$ & $83.1 \pm 42.9$ & $130 \pm 59.4$ & $84.4 \pm 40.1$ & $142.4 \pm 61.5$ \\
\hline $\mathrm{Zn}$ & $31.8 \pm 17.1$ & $29.0 \pm 16.2$ & $64.8 \pm 53.8$ & $45.4 \pm 24.5$ \\
\hline $\mathrm{Ga}$ & $0.28 \pm 0.19$ & $0.18 \pm 0.11$ & $0.28 \pm 0.32$ & $0.28 \pm 0.2$ \\
\hline As & $0.49 \pm 0.3$ & $0.46 \pm 0.35$ & $0.99 \pm 0.57$ & $1 \pm 0.63$ \\
\hline $\mathrm{Se}$ & $0.05 \pm 0.12$ & $0.03 \pm 0.08$ & $1.11 \pm 0.75$ & $0.99 \pm 0.46$ \\
\hline $\mathrm{Br}$ & $1.21 \pm 0.58$ & $1.02 \pm 0.39$ & $5.46 \pm 1.76$ & $5.1 \pm 1.76$ \\
\hline $\mathrm{Rb}$ & $1.51 \pm 0.47$ & $1.31 \pm 0.65$ & $1.16 \pm 0.37$ & $1.01 \pm 0.3$ \\
\hline $\mathrm{Sr}$ & $5.89 \pm 1.8$ & $5.08 \pm 3.64$ & $2.2 \pm 0.98$ & $1.77 \pm 0.66$ \\
\hline $\mathrm{Zr}$ & $2.88 \pm 1.41$ & $3.32 \pm 1.73$ & $1.34 \pm 0.48$ & $1.81 \pm 0.74$ \\
\hline $\mathrm{Sn}$ & $1.31 \pm 0.53$ & $1.35 \pm 1.18$ & $2.45 \pm 2.02$ & $2.19 \pm 1.32$ \\
\hline $\mathrm{Ba}$ & $17.2 \pm 8.11$ & $18.5 \pm 13.8$ & $11.1 \pm 7.51$ & $9.95 \pm 4.72$ \\
\hline $\mathrm{Pb}$ & $11.9 \pm 6.56$ & $11.04 \pm 6.34$ & $34.3 \pm 21.3$ & $28.5 \pm 21.1$ \\
\hline $\begin{array}{c}\text { Total } \mathrm{PM}_{10}^{\mathrm{c}} \\
\left(\mu \mathrm{g} / \mathrm{m}^{3}\right)\end{array}$ & Morning: $57 \pm 13$ & ening: $55 \pm 19$ & & \\
\hline
\end{tabular}

this may demonstrate, on a preliminary level, trends in the "within-a-day" variation of species concentrations. Ionic species and gravimetrically determined PM were not measured during this campaign due to a technical issue. In Tables 3 and 4, the March 2004 results are presented in a similar manner. The water soluble percentage column represents the ratio of the water soluble, ionic concentration to the respective elemental. Tables $5 \mathrm{a}$ and $5 \mathrm{~b}$ present our campaigns total arithmetic means for elements and ions of major interest, next to respective results of previous studies carried out in Athens, in other cities of Greece and other urban centres of the world.

\subsection{August 2003}

3.1.1 Identification of major elements, distribution in the two fractions and comparison of concentrations with air quality limits

During the August 2003 campaign and throughout the year until the summer of 2004, construction work was taking place in the centre of the city. For a big part of August, traffic in downtown Athens was reduced due to the summer vacations. In relation to that, an increase in the measured BA-PM 10 concentrations was observed towards the end of the month, after the end of the DSFU sampling, upon return of the greatest percent of the population. The meteorological conditions during this sampling period were typical of Athens in the middle of summer and were characterized by low relative humidity (daily mean: $42.7 \%$; $\min : 24.0 \%$ ), high temperatures (daily mean: $30.8^{\circ} \mathrm{C}$; min: $24.1^{\circ} \mathrm{C}$; max: 
Table 3. Morning and evening concentrations $\left(\mathrm{ng} / \mathrm{m}^{3}\right)$ measured in Athinas St., $1.5 \mathrm{~m}$ from the ground level, in March 2004 (Mean \pm Standard Deviation); ${ }^{a}$ Coarse Particulate Matter $\left(\mathrm{PM}_{2.5-10}\right) ;{ }^{\mathrm{b}}$ Fine Particulate Matter $\left(\mathrm{PM}_{2.5}\right) ;{ }^{\mathrm{c}} \mathrm{PM}_{10}$ obtained by use of Beta Attenuation at a height of $5 \mathrm{~m}$ from ground level; ${ }^{\mathrm{d}}$ Gravimetrically obtained, at a height of $1.5 \mathrm{~m}$ from the ground level.

\begin{tabular}{|c|c|c|c|c|c|c|c|c|}
\hline \multicolumn{9}{|c|}{ Athinas St. } \\
\hline & $\begin{array}{l}\mathrm{CPM}^{\mathrm{a}} \\
(n=37)\end{array}$ & & & & $\begin{array}{l}\text { FPM }^{\mathrm{b}} \\
(n=29)\end{array}$ & & & \\
\hline & $\begin{array}{l}\text { Morning } \\
(n=21)\end{array}$ & $\begin{array}{l}\% \text { water } \\
\text { soluble }\end{array}$ & $\begin{array}{l}\text { Evening } \\
(n=16)\end{array}$ & $\begin{array}{l}\% \text { water } \\
\text { soluble }\end{array}$ & $\begin{array}{l}\text { Morning } \\
(n=18)\end{array}$ & $\begin{array}{l}\% \text { water } \\
\text { soluble }\end{array}$ & $\begin{array}{l}\text { Evening } \\
(n=11)\end{array}$ & $\begin{array}{l}\% \text { water } \\
\text { soluble }\end{array}$ \\
\hline $\mathrm{Al}$ & $671 \pm 519$ & & $655 \pm 359$ & & $372 \pm 450$ & & $305 \pm 257$ & \\
\hline $\mathrm{Si}$ & $1850 \pm 1415$ & & $1808 \pm 993$ & & $702 \pm 975$ & & $587 \pm 534$ & \\
\hline $\mathrm{SO}_{4}^{2-}$ & $1766 \pm 869$ & & $1958 \pm 795$ & & $6821 \pm 3060$ & & $6540 \pm 3438$ & \\
\hline$S^{4}$ & $656 \pm 354$ & & $809 \pm 397$ & & $2876 \pm 1397$ & & $2556 \pm 1093$ & \\
\hline $\mathrm{S}$ as $\mathrm{SO}_{4}^{-2}$ & $586 \pm 290$ & 89 & $653 \pm 265$ & 81 & $2273 \pm 1020$ & 79 & $2180 \pm 1146$ & 85 \\
\hline $\mathrm{Cl}-4$ & $1199 \pm 1160$ & & $1063 \pm 1027$ & & $204 \pm 419$ & & $163 \pm 183$ & \\
\hline $\mathrm{Cl}^{-}$ & $2474 \pm 1220$ & & $2917 \pm 812$ & & $381 \pm 251$ & & $267 \pm 255$ & \\
\hline $\mathrm{K}$ & $424 \pm 273$ & & $411 \pm 195$ & & $296 \pm 240$ & & $245 \pm 154$ & \\
\hline $\mathrm{K}^{+}$ & $177 \pm 68$ & 42 & $197 \pm 150$ & 48 & $145 \pm 66$ & 49 & $145 \pm 57$ & 59 \\
\hline $\mathrm{Ca}$ & $15999 \pm 10731$ & & $15086 \pm 8087$ & & $3545 \pm 2107$ & & $3581 \pm 1588$ & \\
\hline $\mathrm{Ca}^{2+}$ & $12067 \pm 3597$ & 75 & $11272 \pm 3553$ & 75 & $3372 \pm 1193$ & 95 & $3214 \pm 1630$ & 90 \\
\hline $\mathrm{Ti}$ & $115 \pm 97.3$ & & $136 \pm 73.5$ & & $42.6 \pm 50.9$ & & $74.1 \pm 58.4$ & \\
\hline $\mathrm{V}$ & $5.46 \pm 3.96$ & & $6.47 \pm 3.78$ & & $11.1 \pm 11.9$ & & $8.7 \pm 5.9$ & \\
\hline $\mathrm{Cr}$ & $8.56 \pm 5.46$ & & $8.25 \pm 4.17$ & & $2.59 \pm 2.63$ & & $2.76 \pm 1.58$ & \\
\hline $\mathrm{Mn}$ & $18.2 \pm 12.4$ & & $17.5 \pm 10.3$ & & $12.3 \pm 13.0$ & & $13.0 \pm 10.1$ & \\
\hline $\mathrm{Fe}$ & $1090 \pm 743$ & & $948 \pm 472$ & & $447 \pm 467$ & & $374 \pm 229$ & \\
\hline $\mathrm{Ni}$ & $5.46 \pm 5.36$ & & $4.81 \pm 2.29$ & & $4.47 \pm 4.1$ & & $4.33 \pm 3.32$ & \\
\hline $\mathrm{Cu}$ & $37.2 \pm 23.5$ & & $26.3 \pm 10.62$ & & $22.1 \pm 20.8$ & & $17.7 \pm 11.7$ & \\
\hline $\mathrm{Zn}$ & $52.9 \pm 34.5$ & & $75.5 \pm 42.9$ & & $76.4 \pm 68.9$ & & $118 \pm 113$ & \\
\hline $\mathrm{Ga}$ & $0.37 \pm 0.45$ & & $0.21 \pm 0.13$ & & $0.24 \pm 0.2$ & & $0.14 \pm 0.14$ & \\
\hline As & $0.73 \pm 0.47$ & & $0.92 \pm 0.35$ & & $1.58 \pm 0.48$ & & $1.72 \pm 0.3$ & \\
\hline $\mathrm{Se}$ & $0.15 \pm 0.17$ & & $0.16 \pm 0.12$ & & $0.59 \pm 0.49$ & & $0.49 \pm 0.23$ & \\
\hline $\mathrm{Br}$ & $3.99 \pm 2.38$ & & $3.52 \pm 2.11$ & & $7.39 \pm 0.48$ & & $6.73 \pm 3.58$ & \\
\hline $\mathrm{Rb}$ & $1.85 \pm 1.56$ & & $1.83 \pm 0.77$ & & $1.09 \pm 0.88$ & & $0.74 \pm 0.53$ & \\
\hline $\mathrm{Sr}$ & $15.2 \pm 12.2$ & & $22.2 \pm 16.9$ & & $4.93 \pm 5.32$ & & $5.38 \pm 6.98$ & \\
\hline $\mathrm{Zr}$ & $5.08 \pm 4.54$ & & $4.99 \pm 2.65$ & & $2.3 \pm 2.3$ & & $1.44 \pm 0.66$ & \\
\hline Mo & $2.25 \pm 1.43$ & & $1.38 \pm 0.64$ & & $1.87 \pm 1.32$ & & $1.31 \pm 0.61$ & \\
\hline $\mathrm{Sn}$ & $2.75 \pm 2.38$ & & $2.28 \pm 1.43$ & & $5.01 \pm 6.94$ & & $3.03 \pm 2.10$ & \\
\hline $\mathrm{Sb}$ & $4.84 \pm 3.76$ & & $2.86 \pm 1.63$ & & $3.25 \pm 3.26$ & & $1.88 \pm 1.31$ & \\
\hline $\mathrm{Ba}$ & $28.0 \pm 19.9$ & & $38.6 \pm 33.7$ & & $12.4 \pm 12.8$ & & $16.1 \pm 11.08$ & \\
\hline $\mathrm{Pb}$ & $21.4 \pm 23.2$ & & $14.86 \pm 9.86$ & & $53.9 \pm 66.6$ & & $31.0 \pm 15.4$ & \\
\hline $\mathrm{Na}^{+}$ & $835 \pm 464$ & & $735 \pm 580$ & & $294 \pm 242$ & & $235 \pm 87.5$ & \\
\hline $\mathrm{NH}_{4}^{+}$ & $158 \pm 99$ & & $267 \pm 126$ & & $1484 \pm 1246$ & & $1553 \pm 1014$ & \\
\hline $\mathrm{Mg}^{2+}$ & $277 \pm 133$ & & $327 \pm 173$ & & $109.3 \pm 44.8$ & & $110.2 \pm 44.0$ & \\
\hline $\mathrm{NO}_{3}^{-}$ & $2724 \pm 1836$ & & $2238 \pm 1219$ & & $410 \pm 228$ & & $276 \pm 172$ & \\
\hline $\begin{array}{l}\mathrm{PM}^{\mathrm{d}} \\
\left(\mu \mathrm{g} / \mathrm{m}^{3}\right)\end{array}$ & $94.8 \pm 43.1$ & & $65.3 \pm 30.9$ & & $44.7 \pm 32.7$ & & $36.2 \pm 22.2$ & \\
\hline
\end{tabular}

Total $\mathrm{PM}_{10}^{\mathrm{c}} \quad\left(\mathrm{PM}_{10} \mu \mathrm{m}\right)$ Morning: $104.7 \pm 32.7$; Evening: $78.5 \pm 32.3$ $\left(\mu \mathrm{g} / \mathrm{m}^{3}\right)$ 
Table 4. Morning and evening concentrations ( $\mathrm{ng} / \mathrm{m}^{3}$ ) measured in AEDA in March 2004, $24 \mathrm{~m}$ from the ground level (Mean \pm Standard Deviation); ${ }^{\mathrm{a}}$ Coarse Particulate Matter $\left(\mathrm{PM}_{2.5-10}\right) ;{ }^{\mathrm{b}}$ Fine Particulate Matter $\left(\mathrm{PM}_{2.5}\right) ;{ }^{\mathrm{c}}$ Gravimetrically obtained.

\begin{tabular}{|c|c|c|c|c|c|c|c|c|}
\hline \multicolumn{9}{|c|}{ AEDA } \\
\hline & $\begin{array}{l}\mathrm{CPM}^{\mathrm{a}}(n= \\
30)\end{array}$ & & & & $\begin{array}{l}\mathrm{FPM}^{\mathrm{b}}(n= \\
20)\end{array}$ & & & \\
\hline & $\begin{array}{l}\text { Morning } \\
(n=16)\end{array}$ & $\begin{array}{l}\% \text { water } \\
\text { soluble }\end{array}$ & $\begin{array}{l}\text { Evening } \\
(n=14)\end{array}$ & $\begin{array}{l}\% \text { water } \\
\text { soluble }\end{array}$ & $\begin{array}{l}\text { Morning } \\
(n=11)\end{array}$ & $\begin{array}{l}\% \text { water } \\
\text { soluble }\end{array}$ & $\begin{array}{l}\text { Evening } \\
(n=9)\end{array}$ & $\begin{array}{l}\% \text { water } \\
\text { soluble }\end{array}$ \\
\hline $\mathrm{Al}$ & $411 \pm 211$ & & $428 \pm 284$ & & $320 \pm 174$ & & $273 \pm 174$ & \\
\hline $\mathrm{Si}$ & $1080 \pm 557$ & & $1070 \pm 682$ & & $463 \pm 347$ & & $415 \pm 284$ & \\
\hline $\mathrm{SO}_{4}^{2-}$ & $1092 \pm 442$ & & $1215 \pm 577$ & & $7140 \pm 5844$ & & $8086 \pm 7002$ & \\
\hline $\mathrm{S}$ & $476 \pm 166$ & & $535 \pm 442$ & & $3008 \pm 1924$ & & $2820 \pm 1604$ & \\
\hline $\mathrm{S}_{\text {as }} \mathrm{SO}_{4}^{2-}$ & $364 \pm 180$ & 76 & $405 \pm 192$ & 76 & $2380 \pm 1948$ & 79 & $2645 \pm 2334$ & 94 \\
\hline $\mathrm{Cl} \quad 4$ & $563 \pm 691$ & & $880 \pm 685$ & & $137 \pm 24.01$ & & $110 \pm 133$ & \\
\hline $\mathrm{Cl}^{-}$ & $1041 \pm 686$ & & $1436 \pm 1110$ & & $218 \pm 278$ & - & $173 \pm 100$ & \\
\hline K & $234 \pm 115$ & & $245 \pm 148$ & & $291 \pm 139$ & & $307 \pm 152$ & \\
\hline $\mathrm{K}^{+}$ & $151 \pm 185$ & 65 & $165 \pm 156$ & 67 & $73.4 \pm 56.4$ & 25 & $93.7 \pm 55.8$ & 31 \\
\hline $\mathrm{Ca}$ & $8050 \pm 4870$ & & $7007 \pm 4623$ & & $1728 \pm 946$ & & $1641 \pm 706$ & \\
\hline $\mathrm{Ca}^{2+}$ & $5753 \pm 2151$ & 71 & $5491 \pm 2963$ & 78 & $1375 \pm 947$ & 80 & $1270 \pm 624$ & 77 \\
\hline $\mathrm{Ti}$ & $63.2 \pm 38.4$ & & $51.2 \pm 33.7$ & & $26.1 \pm 14.0$ & & $27.9 \pm 18.9$ & \\
\hline $\mathrm{V}$ & $4.07 \pm 2.74$ & & $4.75 \pm 2.85$ & & $9.32 \pm 5.82$ & & $16.42 \pm 8.91$ & \\
\hline $\mathrm{Cr}$ & $7.99 \pm 6.04$ & & $8.34 \pm 6.23$ & & $4.41 \pm 1.85$ & & $2.71 \pm 0.96$ & \\
\hline $\mathrm{Mn}$ & $12.6 \pm 7.34$ & & $13.1 \pm 9.18$ & & $13.7 \pm 4.46$ & & $9.64 \pm 3.8$ & \\
\hline $\mathrm{Fe}$ & $739 \pm 391$ & & $748 \pm 511$ & & $405 \pm 136$ & & $340 \pm 117$ & \\
\hline $\mathrm{Ni}$ & $3.76 \pm 2.47$ & & $3.62 \pm 3.07$ & & $5.06 \pm 3.23$ & & $5.64 \pm 2.21$ & \\
\hline $\mathrm{Cu}$ & $58.5 \pm 36.9$ & & $63.4 \pm 57.6$ & & $58.5 \pm 23.0$ & & $58.7 \pm 32.2$ & \\
\hline $\mathrm{Zn}$ & $36.3 \pm 22.3$ & & $31.63 \pm 21.7$ & & $73.4 \pm 75.5$ & & $53.5 \pm 27.9$ & \\
\hline $\mathrm{Ga}$ & $0.15 \pm 0.19$ & & $0.19 \pm 0.1$ & & $0.12 \pm 0.05$ & & $0.25 \pm 0.11$ & \\
\hline As & $0.45 \pm 0.28$ & & $0.52 \pm 0.35$ & & $0.73 \pm 0.27$ & & $0.57 \pm 0.1$ & \\
\hline $\mathrm{Se}$ & $0.09 \pm 0.07$ & & $0.09 \pm 0.05$ & & $0.63 \pm 0.4$ & & $0.81 \pm 0.33$ & \\
\hline $\mathrm{Br}$ & $2.12 \pm 1.68$ & & $2.67 \pm 1.29$ & & $6.81 \pm 1.84$ & & $7.8 \pm 2.15$ & \\
\hline $\mathrm{Rb}$ & $1.09 \pm 0.52$ & & $1.01 \pm 0.55$ & & $0.82 \pm 0.5$ & & $1.03 \pm 0.63$ & \\
\hline $\mathrm{Sr}$ & $6.95 \pm 3.93$ & & $6.51 \pm 3.49$ & & $2.77 \pm 1.38$ & & $2.45 \pm 1.18$ & \\
\hline $\mathrm{Zr}$ & $3.26 \pm 1.8$ & & $3.82 \pm 2.62$ & & $1.31 \pm 0.5$ & & $1.83 \pm 0.83$ & \\
\hline Mo & $2.61 \pm 1.59$ & & $3.79 \pm 2.12$ & & $4.56 \pm 1.51$ & & $5.17 \pm 3.17$ & \\
\hline $\mathrm{Sn}$ & $1.92 \pm 1.34$ & & $2.55 \pm 2.14$ & & $3.43 \pm 2.65$ & & $6.32 \pm 6.31$ & \\
\hline $\mathrm{Sb}$ & $3.4 \pm 2.58$ & & $5.19 \pm 2.74$ & & $3.1 \pm 1.0$ & & $3.05 \pm 1.78$ & \\
\hline $\mathrm{Ba}$ & $31 \pm 43.8$ & & $21.7 \pm 12.3$ & & $12.2 \pm 7.12$ & & $5.9 \pm 5.2$ & \\
\hline $\mathrm{Pb}$ & $16.6 \pm 13.2$ & & $25.5 \pm 27.69$ & & $37.2 \pm 27.2$ & & $27.2 \pm 89$ & \\
\hline $\mathrm{Na}^{+}$ & $553 \pm 391$ & & $709 \pm 412$ & & $286 \pm 305$ & & $206 \pm 205$ & \\
\hline $\mathrm{NH}_{4}^{+}$ & $126 \pm 123$ & & $198 \pm 167$ & & $1613 \pm 1731$ & & $1660 \pm 1706$ & \\
\hline $\mathrm{Mg}^{2+}$ & $193 \pm 110$ & & $234 \pm 111$ & & $85.7 \pm 74.6$ & & $80.9 \pm 43.8$ & \\
\hline $\mathrm{NO}_{3}^{-}$ & $1751 \pm 1353$ & & $2031 \pm 1421$ & & $350 \pm 408$ & & $168 \pm 129$ & \\
\hline $\begin{array}{l}\mathrm{PM}^{\mathrm{c}} \\
\left(\mu \mathrm{g} / \mathrm{m}^{3}\right)\end{array}$ & $54.4 \pm 25.1$ & & $55.2 \pm 27.4$ & & $33.0 \pm 8.4$ & & $30.1 \pm 11.6$ & \\
\hline
\end{tabular}

$38.1^{\circ} \mathrm{C}$ ) and low wind speed (daily mean: $2.7 \mathrm{~km} / \mathrm{h}$; $\min$ : $0.4 \mathrm{~km} / \mathrm{h})$.

Measured $\mathrm{BAPM}_{10}$ concentrations shown in Table 2 (morning: $57 \pm 13 \mu \mathrm{g} \mathrm{m}^{-3}$; evening: $55 \pm 19 \mu \mathrm{g} \mathrm{m}^{-3}$; min: $38 \mu \mathrm{g} \mathrm{m}^{-3}$; max: $90 \mu \mathrm{g} \mathrm{m}^{-3}$ ) are very close to the gravimetrically obtained ones reported for June-July of the same year, by another study (Sillanpää et al., 2006) and signify the aerosol pollution problem in GAA, which has already been identified in other studies (Chaloulakou et al., 2003; Grivas et al., 2004; Manalis et al., 2005). Mean daily $\mathrm{PM}_{10}$ values on the site exceeded on 11 days during the campaign the E.U. imposed 24-h limit of $50 \mu \mathrm{g} \mathrm{m}^{-3}$, which is not to be exceeded more than 35 times a year (limit was to be met by January 2005). 
Table 5a. Present study in comparison with literature values. Concentrations are shown in $\mathrm{ng} / \mathrm{m}^{3}$ unless otherwise stated. Sampling heights above ground are shown where declared. Date next to sampling location declares year of measurements, while date in parenthesis declares year of publication; (el): elemental concentration; (i): ionic concentration.

\begin{tabular}{|c|c|c|c|c|c|c|c|c|c|c|}
\hline $\begin{array}{l}\text { Element, } \\
\text { (ng/m3) }\end{array}$ & $\begin{array}{l}\text { Current Study; } \\
\text { Athinas St., } \\
\text { August 2003, } \\
(1.5 \mathrm{~m})\end{array}$ & $\begin{array}{l}\text { Current Study; } \\
\text { Athinas St., } \\
\text { March 2004, } \\
(1.5 \mathrm{~m})\end{array}$ & $\begin{array}{l}\text { Current Study; } \\
\text { AEDA, March } \\
2004, \\
(25 \mathrm{~m})\end{array}$ & $\begin{array}{l}\text { Thes/niki } \\
\text { '99-'00 } \\
\text { (Samara and } \\
\text { Voutsa, 2004) } \\
(3.5 \mathrm{~m})\end{array}$ & $\begin{array}{l}\text { Madrid } \\
\text { 1999-'00 } \\
\text { (Artunano } \\
\text { et al., 2003) }\end{array}$ & $\begin{array}{l}\text { Barcelona } \\
\text { '99-'00 } \\
\text { (Querol } \\
\text { et al., 2001) }\end{array}$ & $\begin{array}{l}\text { Milan, Dec } \\
\text { '97-Sep '98 } \\
\text { (Marcazzan } \\
\text { et al., 2001), } \\
\text { winter;summer } \\
(3 \mathrm{~m})\end{array}$ & $\begin{array}{l}\text { Helsinki } \\
\text { '96-97 } \\
\text { (Pakkanen } \\
\text { et al., 2001) }\end{array}$ & $\begin{array}{l}\text { Vienna } \\
\text { '99-'00 } \\
\text { (Puxbaum } \\
\text { et al., 2004), } \\
(3.4 \mathrm{~m})\end{array}$ & $\begin{array}{l}\text { Budapest } \\
\text { 1996, 1998-99, } \\
\text { (Salma et al., } \\
\text { 2001) }\end{array}$ \\
\hline $\mathrm{CPM}\left(\mu \mathrm{g} / \mathrm{m}^{3}\right)$ & & 80,1 & 54,8 & 39,7 & 47,7 & 49,8 & $110 ; 74$ & 13,0 & 8,9 & \\
\hline $\mathrm{Ca} / \mathrm{Ca}^{2+}$ & $5264(\mathrm{el})$ & 15543(el)/11670(i) & $7529(\mathrm{el}) / 5622(\mathrm{i})$ & & 1920 & 2250 & $1550 ; 1280$ & $480(\mathrm{el}) / 350(\mathrm{i})$ & 540 & $610 ; 2780$ \\
\hline $\mathrm{Fe}$ & 699 & 1019 & 744 & 257 & 1930 & 890 & $2440 ; 1230$ & 520 & 146 & $300 ; 2000$ \\
\hline $\mathrm{Al}$ & 559 & 663 & 420 & & 1820 (as $\mathrm{Al} 2 \mathrm{O} 3)$ & 1220 (as Al2O3) & $1560 ; 1280$ & 520 & & $360 ; 1400$ \\
\hline $\mathrm{Cl} / \mathrm{Cl}-$ & 122(el) & 1131(el)/2696(i) & 722(el)/1239(i) & & $380(\mathrm{el})$ & $1100(\mathrm{el})$ & & 240(i) & $100(\mathrm{el})$ & $50 ; 180$ \\
\hline $\mathrm{K} / \mathrm{K}+$ & $305(\mathrm{el})$ & $418(\mathrm{el}) / 187(\mathrm{i})$ & $240(\mathrm{el}) / 158(\mathrm{i})$ & & $390(\mathrm{el})$ & $560(\mathrm{el})$ & $660 ; 300$ & $200(\mathrm{el}) / 30(\mathrm{i})$ & $80(\mathrm{el})$ & $160 ; 460$ \\
\hline $\mathrm{Zn}$ & 32 & 64 & 34 & 25 & 93 & 250 & $285 ; 140$ & 7.9 & 13 & $10 ; 60$ \\
\hline S & 323 & 733 & 506 & & & & $4100 ; 2640$ & & & $280 ; 890$ \\
\hline $\mathrm{Si}$ & 1287 & 1829 & 1075 & & 4560 (as $\mathrm{SiO} 2)$ & 2960 (as $\mathrm{SiO} 2)$ & $4390 ; 3930$ & & & $990 ; 3820$ \\
\hline $\mathrm{Pb}$ & 11 & 18 & 21 & 15 & 118 & 149 & $310 ; 120$ & 2 & 5.1 & $4 ; 40$ \\
\hline As & 0.48 & 0.83 & 0.49 & & & & & 0.21 & 0.28 & \\
\hline $\mathrm{Cr}$ & 4.69 & 8.41 & 8.17 & 1.36 & 9 & 6 & $14 ;<10$ & & 0.39 & \\
\hline $\mathrm{Ni}$ & 3.94 & 5.14 & 3.69 & 1.4 & 4 & 7 & $10 ;<6$ & 0.79 & 0.4 & \\
\hline $\mathrm{Cu}$ & 107 & 31.8 & 61.0 & 99.9 & 112 & 74 & $90 ; 45$ & 6.2 & 7.9 & \\
\hline Mn & 12.0 & 17.9 & 12.9 & 9.08 & & 24 & & & 4.3 & \\
\hline $\mathrm{v}$ & 5.21 & 5.97 & 4.41 & 2.96 & & 13 & & & 0.53 & \\
\hline $\mathrm{SO}_{4}^{-2}$ & & 1862 & 1154 & & 4380 & 6750 & & 180 & 410 & \\
\hline $\mathrm{NO}_{3}^{-}$ & & 2481 & 1891 & & 2090 & 5720 & & 410 & 1300 & \\
\hline $\mathrm{Mg} / \mathrm{Mg}^{2+}$ & & 302(i) & 213(i) & & $250(\mathrm{el})$ & $290(\mathrm{el})$ & & $130(\mathrm{el}) / 31(\mathrm{i})$ & $60(\mathrm{el})$ & \\
\hline $\mathrm{Na} / \mathrm{Na}^{+}$ & & 785(i) & 631(i) & & $310(\mathrm{el})$ & $940(\mathrm{el})$ & & $300(\mathrm{el}) / 290(\mathrm{i})$ & $120(\mathrm{el})$ & \\
\hline $\mathrm{NH}_{4}^{+}$ & & 192 & 162 & & 1150 & 2710 & & 10 & 300 & \\
\hline
\end{tabular}

Table 5b. Present study in comparison with literature values. Concentrations are shown in $\mathrm{ng} / \mathrm{m}^{3}$ unless otherwise stated. Sampling heights above ground are shown where declared. Date next to sampling location declares year of measurements, while date in parenthesis declares year of publication; (el): elemental concentration; (i): ionic concentration.

\begin{tabular}{|c|c|c|c|c|c|c|c|c|c|c|c|c|c|c|}
\hline $\begin{array}{l}\text { Element, } \\
\text { (ng/m3) }\end{array}$ & $\begin{array}{l}\text { Current } \\
\text { Study; } \\
\text { Athinas St., } \\
\text { August 2003, } \\
(1.5 \mathrm{~m})\end{array}$ & $\begin{array}{l}\text { Current Study; } \\
\text { Athinas St., } \\
\text { March 2004, } \\
(1.5 \mathrm{~m})\end{array}$ & $\begin{array}{l}\text { Current Study; } \\
\text { AEDA, } \\
\text { March 2004, } \\
(25 \mathrm{~m})\end{array}$ & $\begin{array}{l}\text { Athens, } \\
1984 \\
\text { (Valaooras et } \\
\text { al., 1990), } \\
\text { (summer; } \\
\text { winter) }\end{array}$ & $\begin{array}{l}\text { Athens, } 1987 \\
\text { (Scheff and } \\
\text { Valiozis, } \\
\text { 1990), } \\
\text { April-June } \\
(27 \mathrm{~m} \text { ) }\end{array}$ & $\begin{array}{l}\text { Athens, } \\
1995 \text {-'96 } \\
\text { (Thomaidis } \\
\text { et al., 2003), } \\
(25 \mathrm{~m})\end{array}$ & $\begin{array}{l}\text { Thes/niki } \\
\text { '99-'00 } \\
\text { (Samara and } \\
\text { Voutsa, } \\
2004) \\
(3.5 \mathrm{~m})\end{array}$ & $\begin{array}{l}\text { Thes/niki } \\
\text { '89-90 } \\
\text { (Tsitouridou } \\
\text { et al., 1993) } \\
(3 \mathrm{~m})\end{array}$ & $\begin{array}{l}\text { Madrid } \\
1999-' 00 \\
\text { (Artinano et } \\
\text { al., 2003) }\end{array}$ & $\begin{array}{l}\text { Barcelona } \\
\text { '99-'00 } \\
\text { (Querol et } \\
\text { al., 2001) }\end{array}$ & $\begin{array}{l}\text { Milan, Dec } \\
\text { '97-Sep '98 } \\
\text { (Marcazzan } \\
\text { et al., 2001), } \\
\text { winter;summer } \\
\text { (3 m) }\end{array}$ & $\begin{array}{l}\text { Ankara, } \\
\text { Feb and } \\
\text { June } \\
1993 \text {, } \\
\text { (Yatin et al., } \\
\text { 2000) } \\
\text { (2 seasons) }\end{array}$ & $\begin{array}{l}\text { Helsinki } \\
\text { '96-97 } \\
\text { (Pakkanen } \\
\text { et al., } \\
2001 \text { ) }\end{array}$ & $\begin{array}{l}\text { Vienna } \\
\text { '99-'00 } \\
\text { (Puxbaum } \\
\text { et al., } \\
\text { 2004), } \\
(3.4 \mathrm{~m} \text { ) }\end{array}$ \\
\hline $\operatorname{FPM}\left(\mu \mathrm{g} / \mathrm{m}^{3}\right)$ & & 40,5 & 31,6 & & 80,7 & & 66,5 & & 34,1 & 35,0 & $70 ; 51$ & & 11,8 & 21,5 \\
\hline $\mathrm{Ca} / \mathrm{Ca}^{2+}$ & $1390(\mathrm{el})$ & $3563(\mathrm{el}) / 3293(\mathrm{i})$ & $1685(\mathrm{el}) / 1322(\mathrm{i})$ & $\begin{array}{l}1260 \\
770(\text { el) }\end{array}$ & $2610(\mathrm{el})$ & & & 1178 (i) & 660 & 510 & $80 ; 100$ & & $71(\mathrm{el}) / 53(\mathrm{i})$ & 90 \\
\hline $\mathrm{Fe}$ & 368 & 411 & 373 & $490 ; 300$ & 1100 & & 148.4 & & 570 & 260 & $210 ; 170$ & $103 ; 95$ & 96 & 35 \\
\hline $\mathrm{Al}$ & 309 & 339 & 297 & $130 ; 850$ & 408 & & & & 720 (as $\mathrm{Al} 2 \mathrm{O} 3$ ) & 460 (as $\mathrm{Al} 2 \mathrm{O} 3$ ) & $170 ; 250$ & $88 ; 128$ & 59 & \\
\hline $\begin{array}{l}\mathrm{Cl} / \mathrm{Cl}- \\
\mathrm{K} / \mathrm{K}+\end{array}$ & $\begin{array}{l}37(\mathrm{el}) \\
345(\mathrm{el})\end{array}$ & $\begin{array}{l}184(\mathrm{el}) / 324(\mathrm{i}) \\
271(\mathrm{el}) / 145(\mathrm{i})\end{array}$ & $\begin{array}{l}124(\mathrm{el}) / 196(\mathrm{i}) \\
299(\mathrm{el}) / 84(\mathrm{i})\end{array}$ & $\begin{array}{l}75 ; 450(\mathrm{el}) \\
160 ; 280(\mathrm{el})\end{array}$ & $\begin{array}{l}350(\mathrm{el}) \\
428(\mathrm{el})\end{array}$ & & & 2340 (i) & $\begin{array}{l}420 \\
390\end{array}$ & $\begin{array}{l}590(\mathrm{el}) \\
480(\mathrm{el})\end{array}$ & $390 ; 135$ & $\begin{array}{l}42 ; 4.4 \\
128 ; 119\end{array}$ & $\begin{array}{l}43(\mathrm{i}) \\
85(\mathrm{el}) / 67(\mathrm{i})\end{array}$ & $\begin{array}{l}120(\mathrm{el}) \\
190(\mathrm{el})\end{array}$ \\
\hline $\mathrm{s}$ & 4472 & 2716 & 2914 & $970 ; 3100$ & 4180 & & & & & & $2980 ; 2280$ & & & \\
\hline $\begin{array}{l}\mathrm{Si} \\
\mathrm{Zn}\end{array}$ & $\begin{array}{l}450 \\
55\end{array}$ & $\begin{array}{l}645 \\
97\end{array}$ & $\begin{array}{l}439 \\
63\end{array}$ & $\begin{array}{l}410 ; 1110 \\
130 ; 210\end{array}$ & $\begin{array}{l}1210 \\
273\end{array}$ & & 520 & & $\begin{array}{l}1820 \text { (as } \mathrm{SiO} 2) \\
59\end{array}$ & $\begin{array}{l}1050 \text { (as SiO2) } \\
178\end{array}$ & $\begin{array}{l}245 ; 700 \\
145 ; 75\end{array}$ & $20 ; 9.6$ & 14 & 22 \\
\hline $\mathrm{Pb}$ & 31 & 42 & 32 & $610 ; 1100$ & 699 & 190 & 32.2 & & 99 & 130 & $190 ; 85$ & $88 ; 59$ & 5.8 & 17 \\
\hline As & 1.00 & 1.65 & 0.65 & $34 ; 13$ & & 1.9 & & & & & & $2.2 ; 0.63$ & & 0.66 \\
\hline $\mathrm{Cr}$ & 2.42 & 2.68 & 3.56 & & & & 1.1 & & 2 & 6 & $5 ;<10$ & $3.8 ; 2.5$ & & 0.4 \\
\hline $\mathrm{Ni}$ & 5.52 & 4.40 & 5.35 & $21 ; 8.6$ & 12 & 6.5 & 2.36 & & 2 & 6 & $7 ;<6$ & $4.5 ; 1.1$ & 2 & 1.2 \\
\hline $\mathrm{Cu}$ & 113 & 19.9 & 58.6 & $35 ; 26$ & 51 & & 148.5 & & 41 & 52 & $21 ; 14$ & & 3.1 & 3.1 \\
\hline $\mathrm{Mn}$ & 9.11 & 12.7 & 11.7 & 14 & 22 & & 8.57 & & & 14 & & & & 2.4 \\
\hline $\mathrm{v}$ & 13.8 & 9.90 & 12.9 & 6.6 & & & 5.97 & & & 9 & & & & 1.3 \\
\hline $\mathrm{SO}_{4}^{-2}$ & & 6681 & 7613 & & 10400 & & & 9750 & 3770 & 5750 & & $\begin{array}{l}10488 ; \\
1993\end{array}$ & 2500 & 4200 \\
\hline $\mathrm{NO}_{3}^{-}$ & & 343 & 259 & & 2000 & & & 1950 & 1330 & 4030 & & $3298 ; 538$ & 1600 & 2800 \\
\hline $\begin{array}{l}\mathrm{Mg} / \mathrm{Mg}^{2+} \\
\mathrm{Na} / \mathrm{Na}^{+}\end{array}$ & & $\begin{array}{l}110(\mathrm{i}) \\
265(\mathrm{i})\end{array}$ & $\begin{array}{l}83(\mathrm{i}) \\
247(\mathrm{i})\end{array}$ & & $\begin{array}{l}132(\mathrm{el}) \\
369(\mathrm{el})\end{array}$ & & & $\begin{array}{l}70 \\
1143\end{array}$ & $\begin{array}{l}90(\mathrm{el}) \\
130(\mathrm{el})\end{array}$ & $\begin{array}{l}80(\mathrm{el}) \\
230(\mathrm{el})\end{array}$ & & $74 ; 32$ & $\begin{array}{l}25(\mathrm{el}) / 17(\mathrm{i}) \\
170(\mathrm{el}) / 146(\mathrm{i})\end{array}$ & $\begin{array}{l}20 \text { (el) } \\
70 \text { (el) }\end{array}$ \\
\hline $\mathrm{NH}_{4}^{+}$ & & 1519 & 1637 & & & & & 5190 & 1410 & 3180 & & & 1100 & 2200 \\
\hline
\end{tabular}


In August 2003 the dominant element of the CPM is $\mathrm{Ca}$. Other major elements in the fraction include $\mathrm{Al}, \mathrm{Si}$ and Fe. Oxides of these compounds are found in crustal rock; hence a big part of their concentrations is probably related to dust/particle re-suspension. It is worth noting that their evening concentrations do not appear significantly lower compared to the morning ones. Other important components of this fraction are also $\mathrm{S}, \mathrm{K}, \mathrm{Cu}$ and $\mathrm{Zn}$. The aforementioned elements are the most important components of the FPM fraction as well, with $\mathrm{S}$ being, in this case, the predominant element. The biggest part of $\mathrm{Ca}, \mathrm{Al}, \mathrm{Si}$ and $\mathrm{Fe}$ is found in the CPM, which is in agreement with other studies for the eastern Mediterranean (Graham et al., 2004; Kouyoumdjian and Saliba, 2006). Sulphur and zinc are higher in the FPM, and $\mathrm{Cu}$ is found on similar levels in both fractions. A "trend" of the prevalence of evening over morning values for FPM S and $\mathrm{Cu}$ in both fractions is worth mentioning. Lead is mainly found in FPM and its concentration in Athens has been greatly reduced in the last 20 years, as automotive fuel in Greece does no longer contain lead compounds. Arsenic concentration is almost double and Ni slightly higher in the FPM, compared to the CPM, and their concentrations on the site were also found to be significantly lower than the E.U. proposed mean annual limits of $6 \mathrm{ng} \mathrm{m}^{-3}$ and $20 \mathrm{ng} \mathrm{m}^{-3}$ respectively. Manganese is found on similar levels in both fractions and its concentrations are lower by an order of magnitude compared to the WHO-annual tolerance concentration of $150 \mathrm{ng} \mathrm{m}^{-3}$. Vanadium is found mostly in the FPM with a trend for higher values in the evening and its concentrations have in all cases been by two orders of magnitude, or more, lower than the proposed 24-h threshold of $1 \mu \mathrm{g} \mathrm{m}^{-3}$ (WHO, 2000). Barium and $\mathrm{Cr}$ are found at significantly higher levels in the CPM with the concentrations of the later to have increased in the last decade, as is derived from the current and other recent studies (Manalis et al., 2005).

\subsection{March 2004}

3.2.1 The difference between $1.5 \mathrm{~m}$ and $25 \mathrm{~m}$ above ground for PM-mass and compound concentrations

For the March 2004 campaign gravimetrically measured CPM and FPM mean mass concentrations, calculated on a 12-h basis, are shown in Tables 3 and 4, for Athinas St. and AEDA respectively. For Athinas St. BAPM 10 mass concentrations are also shown in Table 3. In this site at $1.5 \mathrm{~m}$ from the ground, the $\mathrm{PM}_{10}(\mathrm{CPM}+\mathrm{FPM})$ campaign mean concentration was $121 \mu \mathrm{g} \mathrm{m}^{-3}$ (max: $242 \mu \mathrm{g} \mathrm{m}^{-3}$ ), with individual $\mathrm{PM}_{10-2.5}$ and $\mathrm{PM}_{2.5}$ concentrations being 80 and $40.5 \mu \mathrm{g} \mathrm{m}^{-3}$ respectively. In the second site (AEDA), at $25 \mathrm{~m}$ above ground, the respective campaign mean concentration for the $\mathrm{PM}_{10}$ was $86 \mu \mathrm{g} \mathrm{m}^{-3}$ ( $\max : 158 \mu \mathrm{g} \mathrm{m}^{-3}$ ), with individual $\mathrm{PM}_{10-2.5}$ and $\mathrm{PM}_{2.5}$ concentrations being 55 and $32 \mu \mathrm{g} \mathrm{m}^{-3}$ respectively. In Athinas St., at $5 \mathrm{~m}$ above ground, the BAPM 10 daily mean concentration was
$92 \mu \mathrm{g} \mathrm{m}^{-3}$ (daily max: $133 \mu \mathrm{g} \mathrm{m}^{-3}$ ). The meteorological conditions were again typical of the season for the city, with moderate relative humidity (daily mean: 63.4\%; min: 49.2\%; max: $79.1 \%$ ), relatively high temperatures (daily mean: $14.6^{\circ} \mathrm{C}$; $\min : 10.8^{\circ} \mathrm{C}$; $\max : 17.9^{\circ} \mathrm{C}$ ) and low wind speed (daily mean: $3.0 \mathrm{~km} / \mathrm{h}$; $\min : 1.6 \mathrm{~km} / \mathrm{h}$; $\max : 5.2 \mathrm{~km} / \mathrm{h}$ ).

A direct comparison of the Athinas St. and AEDA DSFU$\mathrm{PM}_{10}$ (calculated as the sum: CPM+FPM) carried out on a 12-h resolution basis shows that the $\mathrm{PM}_{10}$ mass concentrations in the breathing zone, at a height of $1.5 \mathrm{~m}$ above ground are significantly higher than the ones at $25 \mathrm{~m}$ above ground (Table 6b and Fig. 4b). Additionally, morning CPM mass concentrations in Athinas St., are approximately $30 \%$ higher than the evening CPM concentrations, while the respective difference for the FPM fraction is approximately $22 \%$. In the same site, roughly $35 \%$ of the particulate mass is found in FPM and 65\% in CPM. Additionally, the morning Athinas St. CPM mass concentrations are approximately $43 \%$ higher than the respective ones in AEDA. The difference for the morning FPM concentrations is approximately 28\%. Corresponding evening differences are $26 \%$ and $17 \%$ for the CPM and FPM respectively.

A series of studies have reported discrepancies between the PM mass concentrations obtained with continuous sampling methods such as TEOM or Beta Attenuation (BA) and filter-sampling with gravimetrical quantification (Allen et al., 1997; Charron et al., 2004). Gravimetrically obtained PM mass concentrations are usually found to be higher than the corresponding concentrations obtained with the continuously monitoring methods. The main reason for this is the warming of the sampling line and filter, which results in the loss of semi-volatile compounds like $\mathrm{NH}_{4} \mathrm{NO}_{3}$ and water. However, it has also been found that the discrepancies in the results obtained with the different methods decrease with increasing ambient temperature and decreasing relative humidity (Charron et al., 2004). In a recent study (Gehrig et al., 2005), comparison of results obtained with Beta Attenuation and filter-samplers for two sites largely affected by mineral dust/soil showed a very close to unity BA-PM $10 /$ Grav-PM 10 ratio (1.02 and 1.06). This confirms that the volatilisation losses, expected to occur due to the warming of the sampling line and filter at approx. $25-30^{\circ} \mathrm{C}$ inside the $\mathrm{BA}$, are not significant for traffic-(re)suspended mineral dust. In our study, in addition to the primary focus of directly comparing gravimetric PM mass concentrations obtained in the two sites, comparison of the PM masses obtained with the different methods and at different heights above ground in Athinas St., is considered applicable due to the following reasons. Firstly, Athinas St. has been a heavily influenced by soil/resuspension site, with widespread construction activity taking place. Secondly, the weather conditions in the region during the campaign were characterized by relatively high temperatures and moderate-to-low relative humidities. Thirdly, as will be further discussed below, the region under study is an ammonium-poor environment where the formation of 
Table 6a. Comparison between gravimetrical DSFU-PM 10 (CPM+FPM), collected at a height of $1.5 \mathrm{~m}$ above ground and BA-PM 10 (obtained by use of Beta Attenuation), at a height of approx. $5 \mathrm{~m}$ from the ground level, in Athinas St., in March 2004.

\begin{tabular}{lll}
\hline & Gravimetrical $\mathrm{PM}_{10}(\mathrm{CPM}+\mathrm{FPM})$ & Beta Attenuation $\mathrm{PM}_{10}$ \\
\hline Mean \pm StDev & $121 \pm 46$ & $92 \pm 33$ \\
Median & 137 & 93 \\
Min & 45 & 38 \\
Max & 242 & 165 \\
\hline
\end{tabular}

Difference is significant at the $99 \%$ level, using student t-test.

Table 6b. Comparison between gravimetrical DSFU-PM 10 (CPM+FPM), collected at a height of $1.5 \mathrm{~m}$ above ground in Athinas St. and the respective gravimetrical $\mathrm{PM}_{10}$, collected at a height of $25 \mathrm{~m}$ from ground level in AEDA, in March 2004.

\begin{tabular}{lll}
\hline & $\begin{array}{l}\text { Gravimetrical } \mathrm{PM}_{10}(\mathrm{CPM}+\mathrm{FPM}), \\
\text { Athinas }\end{array}$ & $\begin{array}{l}\text { Gravimetrical } \text { PM }_{10}(\mathrm{CPM}+\mathrm{FPM}), \\
\text { AEDA }\end{array}$ \\
\hline Mean \pm StDev & $121 \pm 46$ & $86 \pm 35$ \\
& (CPM: $80.1 ;$ FPM: 40.5$)$ & (CPM: 54.8; FPM: 31.6) \\
Median & 137 & 84 \\
Min & 45 & 37 \\
Max & 242 & 158 \\
\hline
\end{tabular}

Difference is significant at the $99 \%$ level, using student t-test.

semi-volatile $\mathrm{NH}_{4} \mathrm{NO}_{3}$ is not favoured. In light of the above, half-hourly $\mathrm{PM}_{10}$ values obtained with $\mathrm{BA}\left(\mathrm{BAPM}_{10}\right)$ were averaged over the respective 12 - $h$ periods corresponding to gravimetrical (DSFU) sampling sessions and the BAPM 10 obtained at $5 \mathrm{~m}$ above ground were found to be significantly lower than the gravimetrical DSFU-PM 10 concentrations obtained at $1.5 \mathrm{~m}$ above ground in the same site. Comparative results are shown in Table 6a. and Fig. 4a. Examination of the morning and evening values in Table 3 , for BAPM 10 and DSFU-PM 10 shows that the morning mass concentrations are generally much higher than the evening ones.

The significant increase in the CPM mass concentrations during the "morning" session in Athinas St. could be attributed to the extensive contribution of construction, traffic and traffic-re-suspended soil/dust and overall human activity (e.g. large numbers of pedestrians). One may argue that this "morning-contribution", which, as discussed below, consists mainly of crust-related species like calcium and aluminosilicate compounds, leads to an overestimation of the discrepancy in mass concentrations between the sites and elevations from ground. However, the examination of: a) the discrepancies of the evening CPM concentrations between the different sites and elevations above ground, b) the respective discrepancies of the morning and evening FPM concentrations, c) the discrepancy between the $\mathrm{PM}_{10}$ at $1.5 \mathrm{~m}$ and at $5 \mathrm{~m}$ from the ground in Athinas St., and d) the discrepancy of the maximum concentrations for the three sampling points (Tables $6 \mathrm{a}$ and $6 \mathrm{~b}$ ), confirms that $\mathrm{PM}_{10}$ concentrations significantly increase closer to the ground, near the "breathing zone". This is in agreement with the findings of another study for Beijing (Chan et al., 2005) and fundamental micrometeorological theory, and demonstrates the need for information on the PM mass concentrations in the "breathing-zone" for the centre of Athens.

The AEDA DSFU-PM 10 mass concentrations are very close to the respective $\mathrm{PM}_{10}$ values reported by studies for the same period (Valavanidis et al., 2006), which also entailed measurements at a height of $25 \mathrm{~m}$ above ground level, in a nearby to AEDA location. Our campaign CPM and FPM mean mass concentrations as well as the distribution of PM mass between the two fractions in AEDA $(\sim 63 \%$ in the CPM and $\sim 37 \%$ in the FPM) are also very close to the ones of the aforementioned study. In addition to the above, the AEDA DSFU-PM $_{10}$ are reasonably close to the results of other recent studies (Chaloulakou et al., 2005; Manalis et al., 2005), for the years 2001-2003, in which measurements were again taken at building-storey levels, at approximately $7 \mathrm{~m}$ above ground, or building-roof levels, approximately $25 \mathrm{~m}$ above ground also in or near the centre of Athens. The above support the conclusion that $\mathrm{PM}_{10}$ concentrations are significantly higher close to the ground, near the "breathing zone", compared to the ones measured at higher elevations, which have been presented for the centre of the city to-date. The differences in mass concentrations between these elevations above ground appear to be significantly higher for the heavier CPM and for daytime hours when overall activity in the centre reaches its peak. 
3.2.2 Identification of major elements and ions, distribution in the two fractions and comparison of concentrations with air quality limits

Following the above findings, individual element and ions concentrations have been examined for similar discrepancies between sampling sites and heights. For both sites, and both fractions, major components of the PM are the same as in the previous campaign, with $\mathrm{Ca}$ and aluminosilicates being predominant in the CPM. In the FPM, in AEDA $S$ is the most abundant element, while near the ground, in Athinas St. site, Ca dominates this fraction also, with concentrations significantly higher than the ones of AEDA. Previous publications have mentioned the existence of great amounts of $\mathrm{Ca}$ in the atmosphere of locations in Greece (Danalatos and Glavas, 1995), and in Athens, in particular (Scheff and Valiozis, 1990; Sillanpää et al., 2006), as well as other eastern Mediterranean sites (Graham et al., 2004; Kouyoumdjian and Saliba, 2006). The CPM concentrations of $\mathrm{Ca}, \mathrm{Al}, \mathrm{Si}$, $\mathrm{Fe}$ and $\mathrm{Ti}$ are higher in Athinas St. A similar phenomenon is observed for the respective FPM concentrations of these compounds, with the exception of Fe and Al for which FPM Athinas St. concentrations do not differ much from the ones in AEDA. It is worth mentioning that, during this campaign, $\mathrm{Cu}$ was almost equally distributed in the two fractions, while its concentrations are lower than in the previous campaign, especially in Athinas St. site. Traffic in Athinas St. might have not been further reduced during the March 2004 campaign, compared to August 2003, hence the Cu seasonal variation has raised questions on the extend to which $\mathrm{Cu}$ in downtown Athens is solely related to traffic and whether significant parts of it have been connected with other emission sources as well. As expected, the greater part of $\mathrm{Pb}$ is found in the FPM fraction and its concentration remains very low compared to the air quality limit and results of older studies. Moreover, in both fractions the $\mathrm{Pb}$ concentration between the two sites does not differ significantly. Nickel concentrations do not differ much between the two sites and are generally equally distributed in the CPM and FPM in Athinas St., while its concentrations appear slightly higher in FPM than in CPM in the AEDA site. Arsenic concentrations, on the other hand, appear higher in Athinas St., while in both sites FPM values prevail over CPM. However, both As and $\mathrm{Ni}$ are found in concentrations much lower than the air quality limits, mentioned earlier. $\mathrm{Mn}$ is another compound found in higher concentrations in Athinas St. Its CPM values are higher than in the respective FPM in Athinas St., while in AEDA it is equally distributed in both fractions. Its levels remain at low levels compared to the pre-mentioned air quality threshold. FPM V is again prevalent over CPM; AEDA values are higher than Athinas St. in the first fraction, while the reverse happens for the latter fraction. $\mathrm{Ba}$ and $\mathrm{Cr}$ concentrations are higher in the CPM than in the FPM with their values being similar in the two sites. A slight difference between the two sites, though, is that in Athinas, a trend for their morning values to be higher than their evening values is observed, while in AEDA the reverse is true, with the exception of CPM Cr for which morning and evening values are almost equal.

\subsubsection{March 2004 - ionic concentrations}

A known issue when sampling particulate matter for latter analysis of ionic species is the sampling artefacts which may occur, particularly for ammonium, sulfate, nitrate and chloride. The ambient molar particulate ratio of $\mathrm{NH}_{4}^{+} / \mathrm{SO}_{4}^{2-}$ has been found to be less than $1.5\left(\mathrm{NH}_{4}^{+} / \mathrm{SO}_{4}^{2-} \leq 1.35\right)$, which, as will be further discussed below, is indicative of an ammonium-poor atmosphere, where formation and consequent evaporation of $\mathrm{NH}_{4} \mathrm{NO}_{3}$ is not favoured (Seinfeld and Pandis, 1998; Pathak et al., 2004; Trebs et al., 2005). The absorption of $\mathrm{SO}_{2}$ on particles collected on filters, which could lead to sulfate artefacts, has been found to be negligible for ammonium poor environments (Pathak et al., 2004). This is considered applicable in the present study, given the moderate-to-low $\mathrm{SO}_{2}$ concentrations obtained at two sites nearest to ours during the March campaign $\left(29 \mu \mathrm{g} \mathrm{m}^{-3}\right.$ in Patision and $22 \mu \mathrm{g} \mathrm{m}^{-3}$ in Aristotelous; PERPA; Hellenic Ministry of Environment). Furthermore, the reaction of CPM $\mathrm{CaCO}_{3}$, with $\mathrm{HNO}_{3}$ and $\mathrm{H}_{2} \mathrm{SO}_{4}$ predominates over the reactions of particulate $\mathrm{NaCl}$ with $\mathrm{HNO}_{3}$ and $\mathrm{H}_{2} \mathrm{SO}_{4}$. As a result of this, chloride depletion, via formation of $\mathrm{HCl}$ and evaporation to the gaseous phase, is negligible for the CPM fraction, where the largest percentages of particulate $\mathrm{Cl}^{-}$and $\mathrm{Na}^{+}$have been observed and $\mathrm{Ca}^{2+}$ is by far the most predominant ion. Their nano-equivalent ratio has been found to range between $1.6 \leq \mathrm{Cl}^{-} / \mathrm{Na}^{+} \leq 2.2$ for the two sites, supporting the above conclusion. Chloride depletion was found to occur in the fine fraction, and the percentage of depleted FPM- $\mathrm{Cl}^{-}$, calculated in accordance to (Holland, 1984; Yao et al., 2001), has been found to be approximately $33 \%$ for Athinas St. and 55\% for the AEDA site. However, on three sampling occasions when the concentrations of the rest of the species were not especially high, high FPM concentrations of $\mathrm{Cl}^{-}$and $\mathrm{Na}^{+}$were obtained, with a molar ratio of $\mathrm{Cl}^{-} / \mathrm{Na}^{+}$ well above 1 . The retrospective determination of the origin of those high values is beyond the scope of the current study.

Like with its elemental form, $\mathrm{Ca}^{2+}$ is predominantly found in the CPM, in which it is by far the most abundant element. A relatively big percentage of it is probably found as $\mathrm{CaCO}_{3}$ due to the calcitic nature of the crustal rock in Greece which is supported by the results of a recent study (Sillanpää et al., 2005). Similar with $\mathrm{Ca}^{2+}$ unimodal distribution is observed for $\mathrm{NO}_{3}^{-}, \mathrm{Cl}^{-}$and $\mathrm{Na}^{+}$, as they are mainly found in the CPM. For all of these ions, Athinas St. concentrations are higher than AEDA, for both fractions with the exception of FPM Na${ }^{+}$for which concentrations in the two sites are relatively close. The dominant compound in FPM is $\mathrm{SO}_{4}^{2-}$, which in this fraction is found in slightly higher concentrations in AEDA. At the same time Athinas St. CPM SO${ }_{4}^{2-}$ 


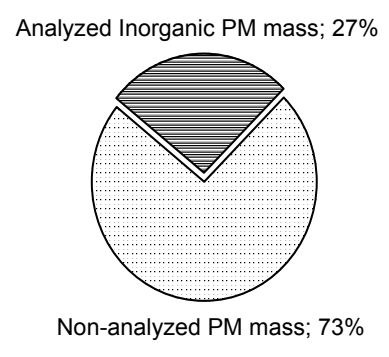

CPM, Morning $(\boldsymbol{n}=\mathbf{2 1})$

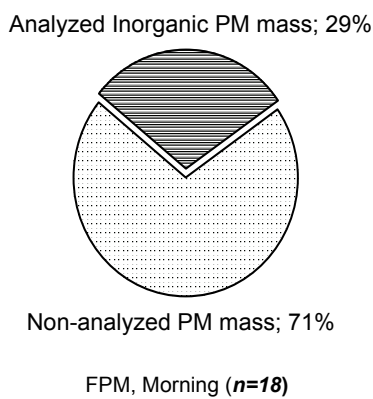

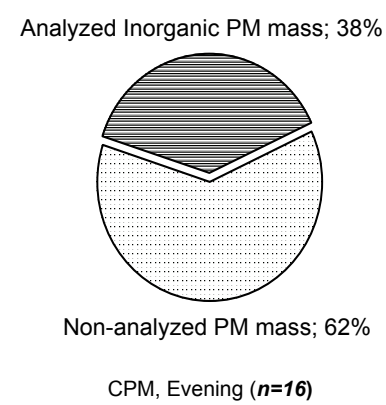

Analyzed Inorganic PM mass; 40\%

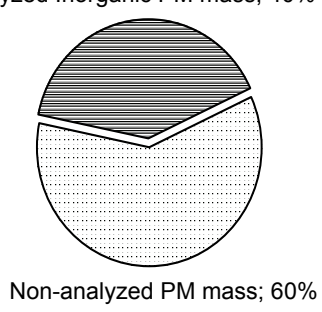

FPM, Evening $(\boldsymbol{n}=11)$

Fig. 2. Total analyzed inorganic versus total collected $\mathrm{PM}_{10}$ mass concentration, in the FPM and CPM fractions, for morning and evening, in Athinas St. site, at $1.5 \mathrm{~m}$ above ground, in March 2004.

is more than the respective in AEDA. The most of $\mathrm{NH}_{4}^{+}$is found in the FPM fraction with AEDA values being slightly higher than the ones of Athinas St. A tendency for slightly higher concentrations of the compound in the evening for both fractions is also observed. Lastly, Athinas $\mathrm{St}^{-\mathrm{Cl}^{-}}$is in both fractions higher than the respective in AEDA with CPM values being more than double in the first site compared to the second.

The ratio of water soluble (ionic) concentration over the total elemental concentration has been calculated. Slightly higher than $70 \%$ of the CPM Ca appears to be water-soluble in both sites. In the FPM, this percentage for Athinas St is near $90 \%$ while in AEDA it appears lower, around $80 \%$. The greatest percentage of $\mathrm{S}$ is also water-soluble in both sites and both fractions, with the CPM ratios ranging from $75 \%$ to $85 \%$ and the FPM ones from $79 \%$ to $94 \%$. Similar findings have been reported for other locations in the eastern Mediterranean (Graham et al., 2004). In Athinas St., for both fractions, on average $50 \%$ of $\mathrm{K}^{+}$appears to be water soluble, while in AEDA the respective percentages are $60 \%$ in the CPM and $30 \%$ in the FPM.

3.3 Seasonal variation; comparison between August 2003 and March 2004; comparison with other studies in Athens and in other cities

A direct comparison of Athinas St. campaign mean concentrations is shown in Table 5a and $5 \mathrm{~b}$. For most major elements and ions, the March 2004 concentrations in Athinas
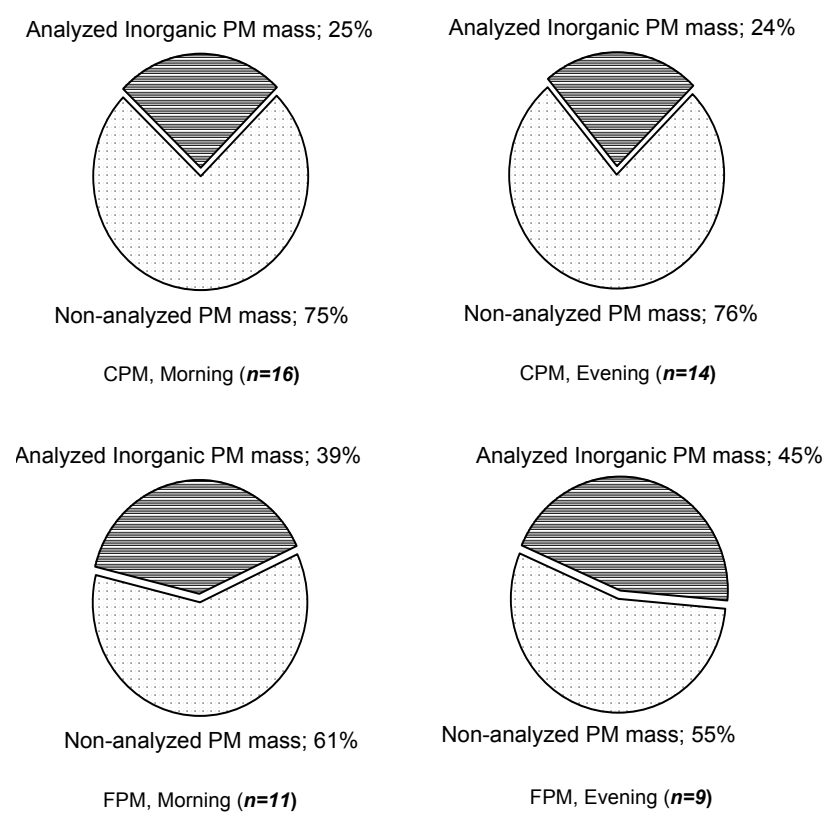

Fig. 3. Total analyzed inorganic versus total collected $\mathrm{PM}_{10}$ mass concentration, in the FPM and CPM fractions, for morning and evening, in AEDA site, at $25 \mathrm{~m}$ above ground, in March 2004.

St. appear in both fractions significantly elevated compared to the respective ones of August 2003. For example, Ca concentrations are in both fractions more than double in March compared to August. Two exceptions to the above are observed. The first is $\mathrm{Al}$ for which only a small increase is observed in March, for both fractions. The second is S, the greatest percent of which is found as sulphate, which appears higher in the CPM in March but significantly lower in the FPM compared to August. CPM S, Si, Mn, $\mathrm{Cr}, \mathrm{Pb}$ and $\mathrm{Zn}$ are also higher in March, while, as mentioned above, the reverse is true for $\mathrm{Cu}$. An increase in the concentration of the latter has been recently reported compared to the late 80 's (Manalis et al., 2005). Such an increase was seen in the August campaign, when in Athinas St FPM Cu mean concentration has more than doubled compared to the one reported by Scheff and Valiozis (1990), but not during the March campaign. During the March campaign, AEDA $\mathrm{Cu}$ concentrations were found to be only slightly higher to the ones reported by Scheff and Valiozis (1990). The respective March 2004 Athinas St. concentration was lower than the one obtained in the August 2003 campaign in the same site, lower than the one obtained in AEDA in the same campaign, and lower than the one reported by Scheff and Valiozis (1990). It can thus be derived, that $\mathrm{Cu}$-enrichment sources during the August 2003 were less active or influencing the Athinas St site during March 2004, which is to be further researched. A similar seasonal pattern is observed for higher in August and overall high sulphur concentrations. Significant contribution to the sulphur-concentrations is believed to be due to the 

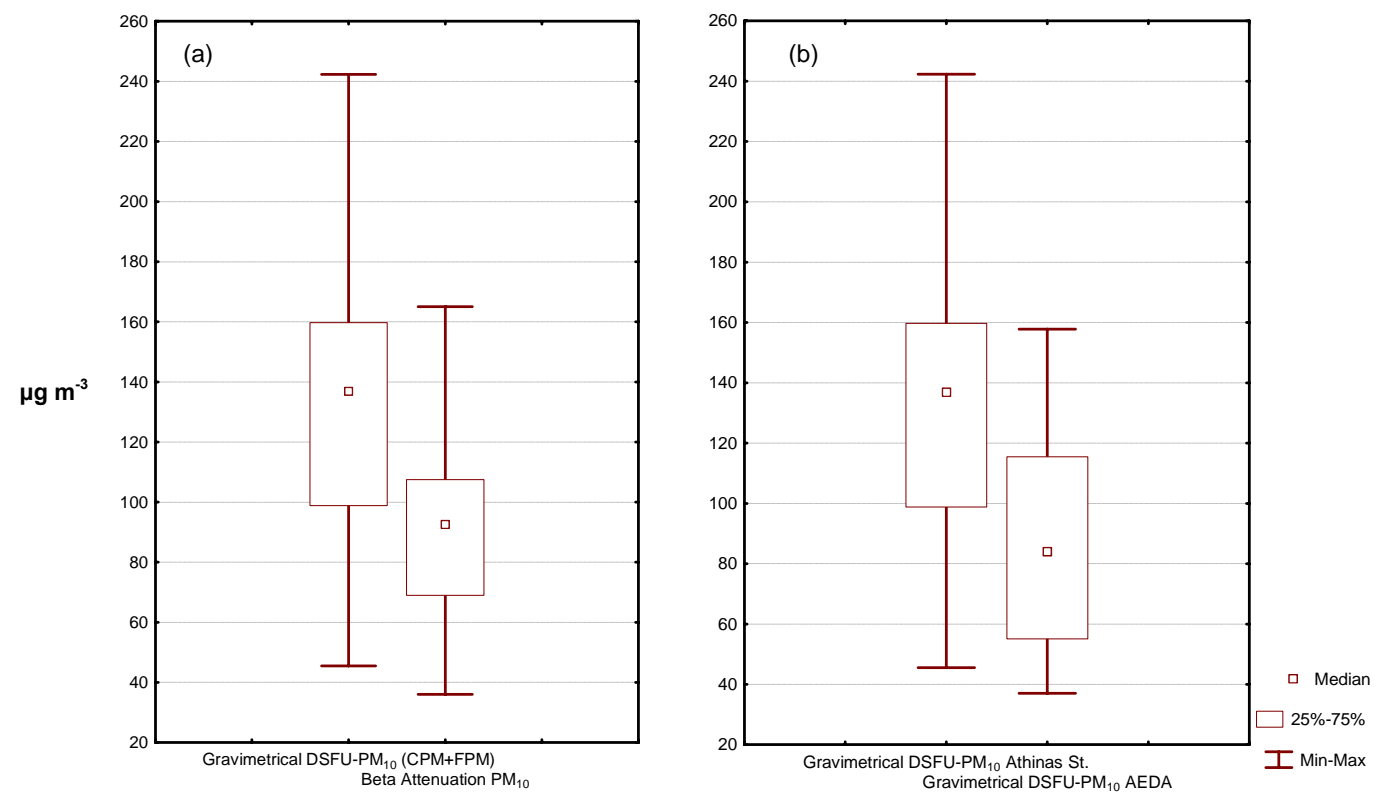

Fig. 4. (a) Comparative graph of the gravimetrical DSFU-PM 10 (calculated as the sum of CPM+FPM) collected at a height of $1.5 \mathrm{~m}$ above ground and BA-PM 10 (obtained by Beta Attenuation) collected at $5 \mathrm{~m}$ above ground, in Athinas St., in March 2004; (b) Comparative graph of the gravimetrical DSFU-PM 10 collected at a height of $1.5 \mathrm{~m}$ above ground in Athinas St., and the respective gravimetrical DSFU-PM 10 at a height of approx. $25 \mathrm{~m}$ above ground in AEDA.

high sulphur-content of diesel for road-transport in Greece (Kalligeros et al., 2001; Commission, 2005), in addition to the major contribution by fossil-fuel combustion for domestic heating during the March 2004. In this study, as well as in other recent studies with similar findings (Manalis et al., 2005), $\mathrm{PM}_{10}$ (CPM+FPM) $\mathrm{Pb}$ is found to be significantly lower than the E.U. imposed annual limit of $500 \mathrm{ng} / \mathrm{m}^{3}$, and lower by an order of magnitude or more, compared to measurements dating a decade back (Thomaidis et al., 2003). On the other hand, in March, CPM Vanadium remains on the same levels with the previous campaign. Similar is the case for the FPM values of the above compounds, as they also appear higher in March 2004, with the aforementioned exception of sulphur. In a similar manner, FPM Cu and V have also been found at higher concentrations during the August 2003 campaign.

In addition to the seasonal variation in the concentrations, in Tables $5 \mathrm{a}$ and $5 \mathrm{~b}$ a comparison of values measured during the current study with the ones of previous studies in Athens and other cities is shown. The CPM Ca concentrations appear significantly higher than the values recently reported for Madrid, Barcelona and Milan which also have elevated calcium concentrations. For other metals of crustal origin such as $\mathrm{Fe}, \mathrm{Al}$ and $\mathrm{Si}$, as well as some of the toxic metals such as $\mathrm{Cr}, \mathrm{Pb}, \mathrm{Zn}$ and $\mathrm{Mn}$, values are on the same levels or lower to the values for the abovementioned cities. Similar is the case for most ionic species in most fractions, with the exception of marine related $\mathrm{Na}+$ and $\mathrm{Cl}-$, which appears in higher concen- trations than Madrid but lower than Barcelona. In general, for most species concentrations in the CPM fraction are generally significantly higher than the ones reported for other cities to the North of Europe, like Vienna, Helsinki and Budapest.

In the FPM, the calcium concentrations are generally close to the ones measured in Athens in the 80's. The August '03 $\mathrm{S}$ concentrations appear very close to the ones presented in an older study (Scheff and Valiozis, 1990), which entailed measurements in the late spring and early summer. In this fraction, most other metals of crustal origin appear in lower concentrations than the ones of the previous studies, and generally close to the ones for the aforementioned Mediterranean cities. FPM $\mathrm{SO}_{4}^{2-}$ appears slightly lower and $\mathrm{NO}_{3}^{-}$ significantly lower than in past studies in Athens (Scheff and Valiozis, 1990), and Thessaloniki, Greece (Tsitouridou and Samara, 1993). The $\mathrm{FPM} \mathrm{SO}_{4}^{2-}$, on the other hand, is slightly higher than the values reported for Madrid, Barcelona and Ankara while the $\mathrm{FPM} \mathrm{NO}_{3}^{-}$appears significantly lower than the respective concentrations for these cities.

\subsection{Analyzed inorganic versus total particulate mass}

In Figs. 2 and 3 the analyzed inorganic mass against the total sampled particulate mass is presented separately for morning and evening, for both sites and both fractions. For the pertinent calculations the mean individual masses of all compounds have been summed up. For the compounds that have 

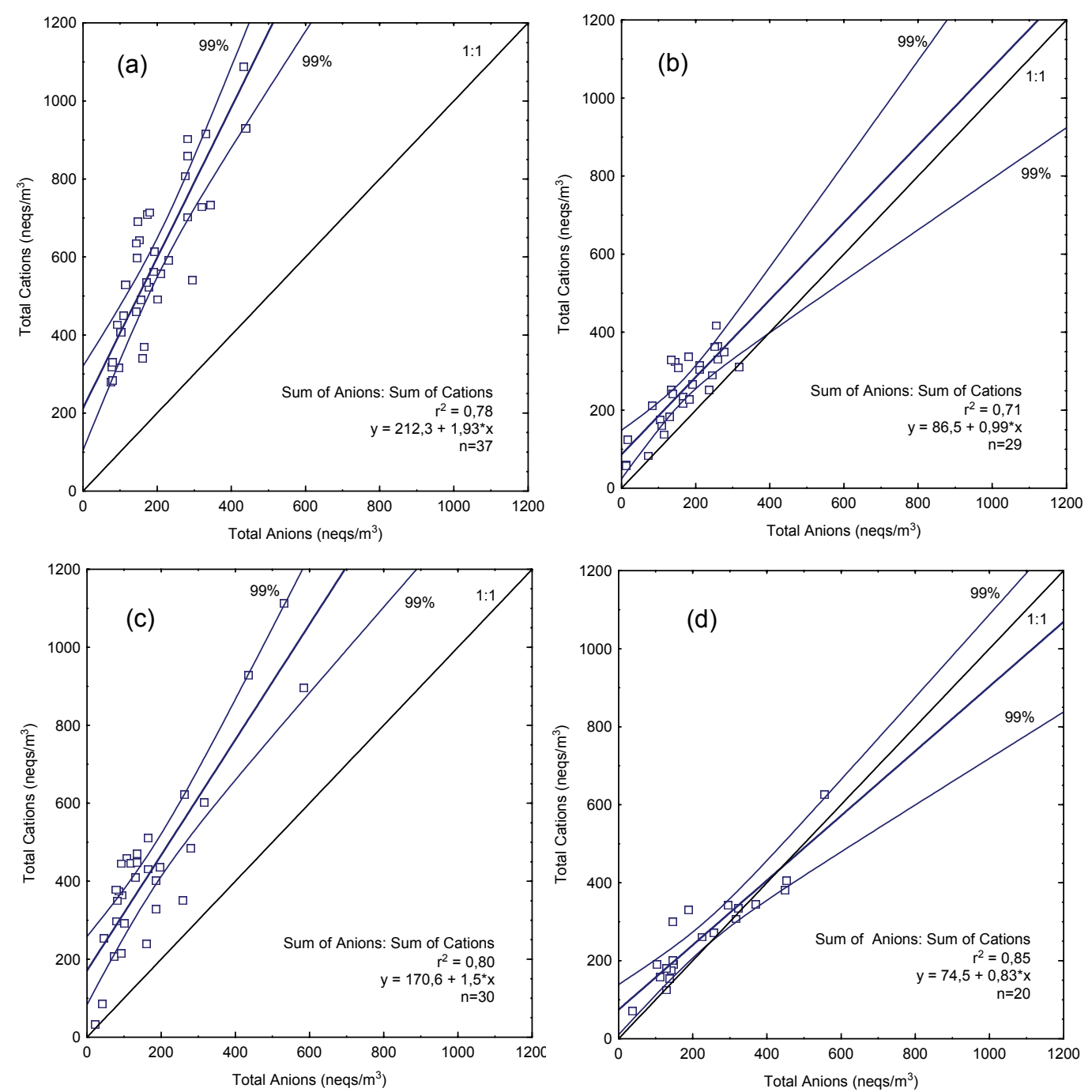

Fig. 5. Ionic balances for both fractions in the two sites. From top left to lower right: (a) Athinas St. CPM, and (b) FPM; (c) AEDA CPM, and (d) FPM.

been measured both in their elemental and ionic form (e.g. $\mathrm{Ca}$ and $\mathrm{Ca}^{2+}$ ), the elemental has been used, with the exception of $\mathrm{Cl} / \mathrm{Cl}^{-}$for which the ionic form has been used, due to the aforementioned issue of losses of $\mathrm{Cl}$ in the PIXE analysis. Moreover, sulphur has been determined in its elemental form and as $\mathrm{SO}_{4}^{2-}$. Due to its higher molecular weight, $\mathrm{SO}_{4}^{2-}$ constitutes higher mass concentrations than sulphur. As a result of this, the ionic $\mathrm{SO}_{4}^{2-}$ mass concentrations have been used in the pie-chart calculations instead of the elemental S. This slightly underestimates the sulphur "contribution" to the slice of known chemical composition in the pies, as it takes into account only the part of sulphur that is found as $\mathrm{SO}_{4}^{2-}$. However, as the ratio $\left[\mathrm{S}_{\text {as }} \mathrm{SO}_{4}^{2-}\right] /[\mathrm{S}]$ has been found to range from $75 \%$ to $95 \%$ and given the approximate character of this calculation, this underestimation is not considered very important.
Analyzed CPM mass ranges from $24 \%$ to $27 \%$ of the total CPM mass, with the exception of Athinas St. in the evening where the respective percent appears significantly higher (38\%). While further examination of the contributing sources is needed, on a preliminary level the elevated values in the evening CPM in Athinas St. may be related to the fact that, while CPM mass is significantly lower than in the morning CPM, the calcium concentrations, both elemental and ionic, remain at very high levels. The analyzed FPM mass ranges from $39 \%$ to $45 \%$ of the total FPM mass, with the exception of Athinas St. in the morning where this percentage appears lower at approximately $29 \%$. Ions such as $\mathrm{CO}_{3}^{-}$which has been recently identified in great amounts in the Athens atmosphere (Sillanpää et al., 2005), $\mathrm{OH}^{-}$, lighter elements that PIXE can not successfully analyze, strongly bound water that remains at the low $\mathrm{RH}$ of the weighing 
Table 7. The correlation coefficients $\left(r^{2}\right)$ between the major inorganic anions and the respective cations for a). Athinas St.; b) for AEDA.

\begin{tabular}{|c|c|c|c|c|c|c|c|c|c|c|c|c|c|c|c|c|c|}
\hline \multicolumn{18}{|l|}{ a) } \\
\hline Athinas St. & $\mathrm{CPM}$ & & & & & & & & & FPM & & & & & & & \\
\hline & $\mathrm{Na}^{+}$ & $\mathrm{NH}_{4}^{+}$ & $\mathrm{K}^{+}$ & $\mathrm{Mg}^{2+}$ & $\mathrm{Ca}^{2+}$ & $\mathrm{Cl}^{-}$ & $\mathrm{SO}_{4}^{2-}$ & $\mathrm{NO}_{3}^{-}$ & & $\mathrm{Na}^{+}$ & $\mathrm{NH}_{4}^{+}$ & $\mathrm{K}^{+}$ & $\mathrm{Mg}^{2+}$ & $\mathrm{Ca}^{2+}$ & $\mathrm{Cl}^{-}$ & $\mathrm{SO}_{4}^{2-}$ & $\mathrm{NO}_{3}^{-}$ \\
\hline $\mathrm{Na}^{+}$ & 1 & & & & & & & & $\mathrm{Na}^{+}$ & 1 & & & & & & & \\
\hline $\mathrm{NH}_{4}^{+}$ & .45 & 1 & & & & & & & $\mathrm{NH}_{4}^{+}$ & .32 & 1 & & & & & & \\
\hline $\mathrm{K}^{+^{4}}$ & .18 & .01 & 1 & & & & & & $\mathrm{~K}^{+{ }^{+}}$ & .16 & .44 & 1 & & & & & \\
\hline $\mathrm{Mg}^{2+}$ & .81 & .36 & .00 & 1 & & & & & $\mathrm{Mg}^{2+}$ & .76 & .24 & .02 & 1 & & & & \\
\hline $\mathrm{Ca}^{2+}$ & .19 & .00 & .06 & .44 & 1 & & & & $\mathrm{Ca}^{2+}$ & .07 & .12 & .00 & .35 & 1 & & & \\
\hline $\mathrm{Cl}^{-}$ & .92 & .32 & .56 & .74 & .22 & 1 & & & $\mathrm{Cl}^{-}$ & .76 & .31 & .23 & .56 & .09 & 1 & & \\
\hline $\mathrm{SO}_{4}^{2-}$ & .24 & .20 & .17 & .14 & .53 & .04 & 1 & & $\mathrm{SO}_{4}^{2-}$ & .16 & .72 & .36 & .05 & .19 & .20 & 1 & \\
\hline $\mathrm{NO}_{3}^{-}$ & .04 & .00 & .00 & .13 & .47 & .05 & .44 & 1 & $\mathrm{NO}_{3}^{-}$ & .35 & .19 & .05 & .54 & .42 & .08 & .02 & 1 \\
\hline
\end{tabular}

b)

\begin{tabular}{|c|c|c|c|c|c|c|c|c|c|c|c|c|c|c|c|c|c|}
\hline AEDA & CPM & & & & & & & & & FPM & & & & & & & \\
\hline & $\mathrm{Na}^{+}$ & $\mathrm{NH}_{4}^{+}$ & $\mathrm{K}^{+}$ & $\mathrm{Mg}^{2+}$ & $\mathrm{Ca}^{2+}$ & $\mathrm{Cl}^{-}$ & $\mathrm{SO}_{4}^{2-}$ & $\mathrm{NO}_{3}^{-}$ & & $\mathrm{Na}^{+}$ & $\mathrm{NH}_{4}^{+}$ & $\mathrm{K}^{+}$ & $\mathrm{Mg}^{2+}$ & $\mathrm{Ca}^{2+}$ & $\mathrm{Cl}^{-}$ & $\mathrm{SO}_{4}^{2-}$ & $\mathrm{NO}_{3}^{-}$ \\
\hline $\mathrm{Na}^{+}$ & 1 & & & & & & & & $\mathrm{Na}^{+}$ & 1 & & & & & & & \\
\hline $\mathrm{NH}_{4}^{+}$ & .74 & 1 & & & & & & & $\mathrm{NH}_{4}^{+}$ & .00 & 1 & & & & & & \\
\hline $\mathrm{K}^{+{ }^{4}}$ & .01 & .00 & 1 & & & & & & $\mathrm{~K}^{+}$ & .00 & .00 & 1 & & & & & \\
\hline $\mathrm{Mg}^{2+}$ & .85 & .51 & .00 & 1 & & & & & $\mathrm{Mg}^{2+}$ & .64 & .02 & .00 & 1 & & & & \\
\hline $\mathrm{Ca}^{2+}$ & .01 & $\begin{array}{l}.01 \\
.09\end{array}$ & .00 & .23 & 1 & & & & $\mathrm{Ca}^{2+}$ & .22 & .00 & .07 & .38 & 1 & & & \\
\hline $\mathrm{Cl}^{-}$ & .94 & .66 & .10 & .92 & .01 & 1 & & & $\mathrm{Cl}^{-}$ & .85 & .02 & .00 & .92 & 28 & 1 & & \\
\hline $\mathrm{SO}_{4}^{2-}$ & .25 & .44 & .00 & .32 & .54 & .20 & 1 & & $\mathrm{SO}_{4}^{2-}$ & .04 & .80 & .11 & .14 & .21 & .12 & 1 & \\
\hline $\mathrm{NO}_{3}^{-}$ & .00 & .00 & .01 & .03 & .45 & .00 & .18 & 1 & $\mathrm{NO}_{3}^{-}$ & .34 & .08 & .01 & .77 & .47 & .40 & .30 & 1 \\
\hline
\end{tabular}

chamber (Harrison et al., 2003) other mineral compounds (including bound oxygen and nitrogen), as well as carbon, organics and polymers, are believed to constitute the biggest percentage of the unknown part of the PM. According to recent studies (D'Alessandro et al., 2003), these compounds usually constitute more than $50 \%$ of the urban aerosol mass.

3.5 Ionic balances and neutralization of atmospheric acidity

Strong correlations between the nano-equivalent sum of cations $\left(\mathrm{Na}^{+}, \mathrm{NH}_{4}^{+}, \mathrm{K}^{+}, \mathrm{Mg}^{2+}, \mathrm{Ca}^{2+}\right)$ and the nanoequivalent sum of anions $\left(\mathrm{Cl}^{-}, \mathrm{NO}_{3}^{-}, \mathrm{SO}_{4}^{2-}\right)$ have been found for both sites and both fractions (Fig. 5). However, as can be seen in the ionic balance plots, a clear deficit of anions is observed in the CPM while in the FPM the anion/cation ratio is close to 1 . The anionic deficit in the CPM is probably due to the carbonates, hydroxides, as well as carboxylic acids which are probably bound with the excessive amounts of $\mathrm{Ca}^{2+}$ and are not analyzable by our method.

Atmospheric ammonia, in its $\mathrm{NH}_{4}^{+}$form, is predominantly found in FPM whilst the CPM is $\mathrm{NH}_{4}^{+}$-poor. As has been previously stated, the ambient molar particulate $\mathrm{NH}_{4}^{+} / \mathrm{SO}_{4}^{2-}$ ratio has been found to be less than 1.5 , which is indicative of an ammonium-poor aerosol system, where the existing $\mathrm{NH}_{4}^{+}$ is insufficient to completely neutralize the available sulphuric acid and the formation of $\mathrm{NH}_{4} \mathrm{NO}_{3}$ is not favoured (Seinfeld and Pandis, 1998 and references therein; Pathak and
Chan, 2005; Trebs et al., 2005). The correlations between $\mathrm{NH}_{4}^{+}$and $\mathrm{SO}_{4}^{2-}$ (Tables $7 \mathrm{a}$ and $\mathrm{b}$ ) indeed suggest that in the fine particles, $\mathrm{NH}_{4}^{+}$is an important neutralizing agent of sulphuric acid forming salts like $\mathrm{NH}_{4} \mathrm{HSO}_{4},\left(\mathrm{NH}_{4}\right)_{3}\left(\mathrm{SO}_{4}\right)_{2}$ and $\left(\mathrm{NH}_{4}\right)_{2} \mathrm{SO}_{4}$. In addition to this, and as expected, the respective correlations for $\mathrm{NH}_{4}^{+}$and $\mathrm{NO}_{3}^{-}$are low, in agreement with the prediction that formation of $\mathrm{NH}_{4} \mathrm{NO}_{3}$ is not expected to occur to a significant extend. The relatively little $\mathrm{NO}_{3}^{-}$in the fine fraction, correlates well with $\mathrm{Mg}^{2+}$ and relatively well with $\mathrm{Ca}^{2+}$ which is indicative of formation of salts like $\mathrm{Mg}\left(\mathrm{NO}_{3}\right)_{2}$ and $\mathrm{Ca}\left(\mathrm{NO}_{3}\right)_{2}$.

Water-soluble ionic calcium is by far the most abundant specie in the CPM fraction. Hence, the good correlation between $\mathrm{CPM} \mathrm{Ca}{ }^{2+}$ and $\mathrm{CPM} \mathrm{NO}-$ and $\mathrm{SO}_{4}^{2-}$ (Tables $7 \mathrm{a}$ and $b$ ), suggests that calcium-containing aerosols, originating from the calcium-rich soils in the region as well as the quick-lime extensively used in painting facades in Greece, are probably the most important neutralizing species in the coarse fraction, on the surface of which acids like $\mathrm{H}_{2} \mathrm{SO}_{4}$ and $\mathrm{HNO}_{3}$ react to form $\mathrm{Ca}\left(\mathrm{NO}_{3}\right)_{2}$ and $\mathrm{CaSO}_{4}$. Lastly, strong correlations observed between $\mathrm{Na}^{+}$and $\mathrm{Cl}^{-}$and $\mathrm{Mg}^{2+}$ and $\mathrm{Cl}^{-}$, for both fractions, indicate the presence of $\mathrm{NaCl}$ and $\mathrm{MgCl}_{2}$ salts, the biggest percentage of which is found in the coarse fraction. The above are in agreement with aforementioned studies in Greece (Tsitouridou and Samara, 1993; Danalatos and Glavas, 1995; Eleftheriadis et al., 1998), in other locations with similar crustal composition, in the East- 

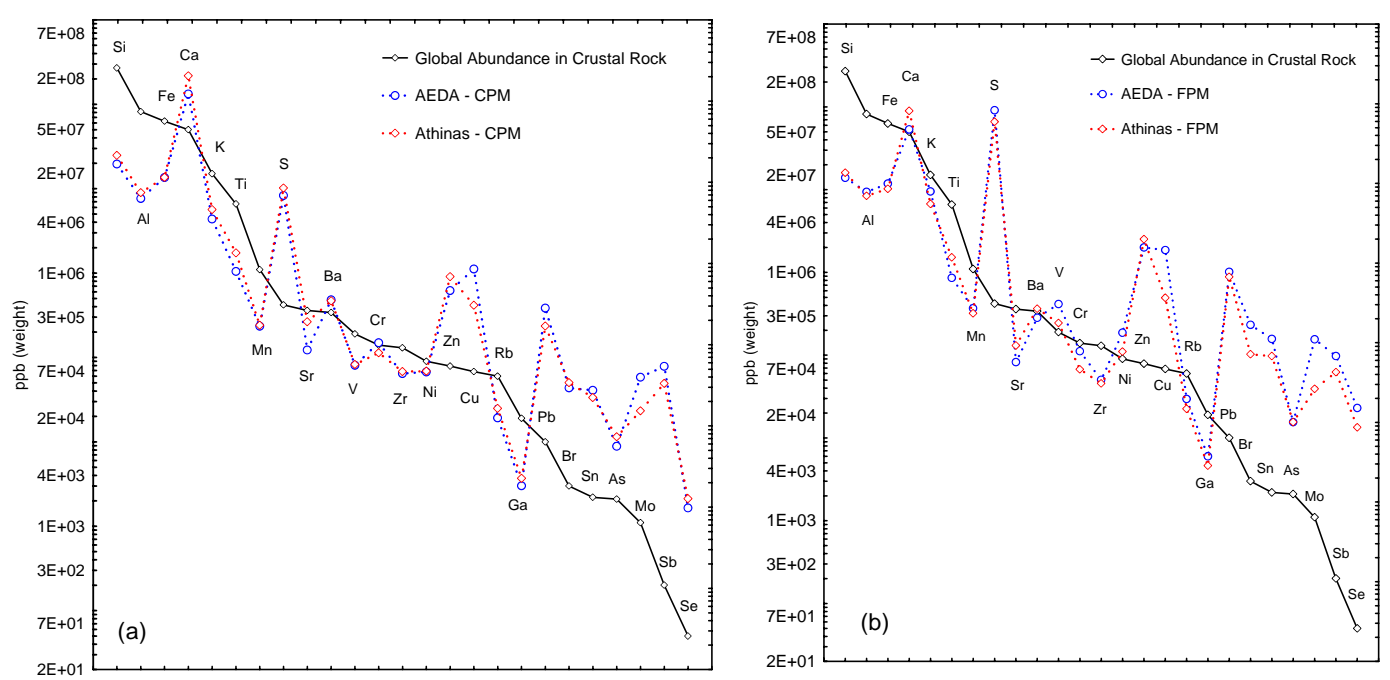

Fig. 6. Elemental chemical composition in the two sites (Athinas St. and AEDA) for (a) CPM and (b) FPM, plotted together with the average global abundance of elements in crustal rock as a reference.
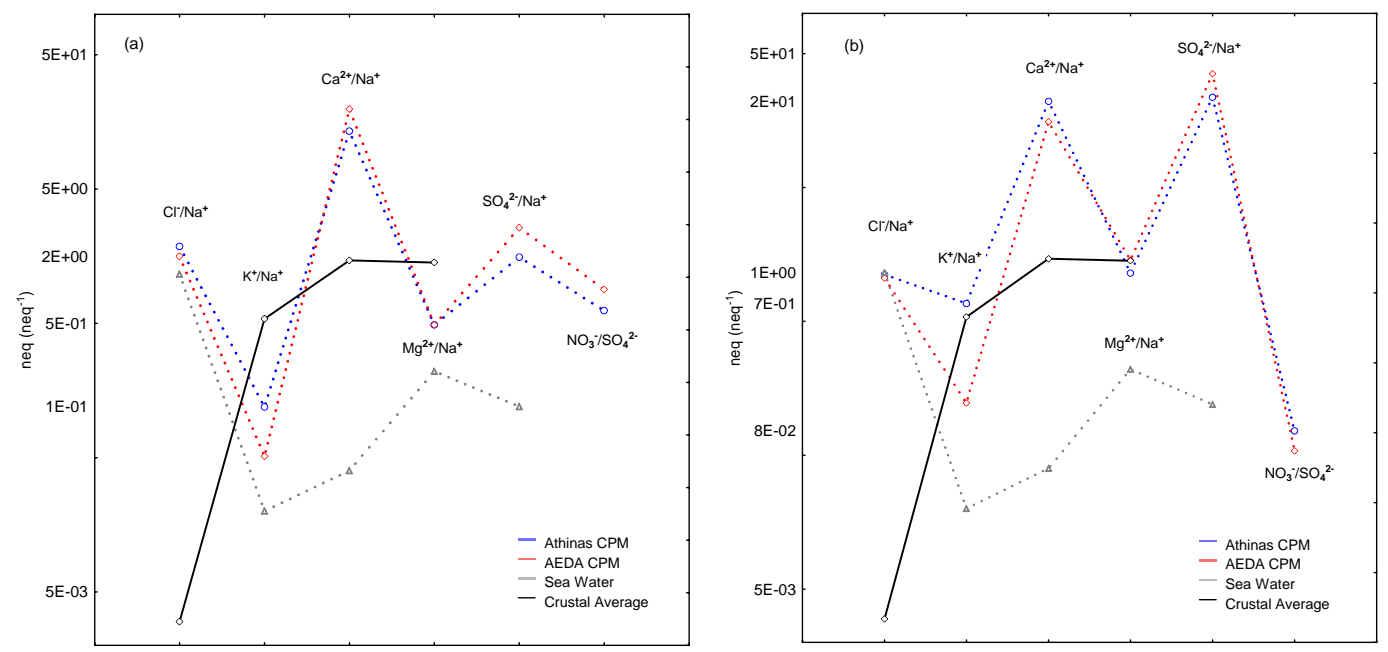

Fig. 7. Ionic concentration ratios in the two sites (Athinas St. and AEDA) for (a) CPM and (b) FPM, plotted together with the sea-water and crustal global averages.

ern Mediterranean (Graham et al., 2004; Kouyoumdjian and Saliba, 2006), as well as in other parts of the world (Davis and Jixian, 2000; Pathak et al., 2004; Xie et al., 2005).

3.6 An attempt to establish the fingerprint of inorganic aerosol pollution

In an attempt to graphically establish the fingerprint of the elemental composition of $\mathrm{PM}_{10}$ aerosol in Athens, we compare our findings with globally averaged crustal composition (Fyfe, 1974; Mason and Moore, 1982; www.webelements. com), in Figs. 6a and b and in Figs. 7a and b. Although quantitative comparisons are not possible since there exist no data on the pristine Athenian $\mathrm{PM}_{10}$ composition, this qualita- tive comparison indicates characteristic elemental and ionic peaks that are attributed to the geochemical characteristics of the area, as well as the nature of human activity. For example, one could note $\mathrm{Ca}$ enrichment and $\mathrm{Ga}, \mathrm{Sr}$ and $\mathrm{Zr}$ deficit. It could also be argued that $\mathrm{S}, \mathrm{Zn}, \mathrm{Cu}, \mathrm{Pb}, \mathrm{Sn}, \mathrm{Mo}$, As and $\mathrm{Sb}$ peaks can be attributed to fossil fuel burning and to traffic related emissions (tyres, catalysts and brakes). The extensive construction activity in the centre of the city at the time has probably contributed to a significant extend, to the aforementioned $\mathrm{Ca}$ enrichment, especially nearer to the ground and the construction works in Athinas St. In addition to this, it is shown that with the exception of $\mathrm{Zn}$, the characteristic automotive traffic/exhaust-emitted elemental peaks are slightly higher for AEDA, which lies nearby an important cross-road 
with high diesel-burning taxi circulation and a nearby dieselburning city-bus station, indicating slightly stronger influence of traffic/vehicle-exhaust pollution for this site. From the respective ionic ratios it can be derived that a big part of $\mathrm{Cl}^{-}$is found as $\mathrm{NaCl}$ originating from sea-spray. In the $\mathrm{CPM}$, an influence by some additional non-marine source of $\mathrm{Cl}^{-}$is probably also present. The $\mathrm{Mg}^{2+} / \mathrm{Na}^{+}$ratio appears very close to the crustal average for the FPM fraction, which might be indicative of dust contributions in this fraction. The same ratio appears close to the sea-water average for the CPM fraction which is in accordance to the aforementioned finding that a big part of CPM Mg${ }^{2+}$ is found as $\mathrm{MgCl}_{2}$ originating from sea spray. The $\mathrm{SO}_{4}^{2-} / \mathrm{Na}^{+}$ratio for both CPM and FPM appears significantly higher than the one of the sea-water which, given the fact that the earth's crust is a negligible source of sulfates (Yaaqub et al., 1991), suggests the existence of anthropogenic sources contributing to $\mathrm{SO}_{4}^{2-}$ concentrations. Lastly, the calcium enrichment is again obvious as the ratio $\mathrm{Ca}^{2+} / \mathrm{Na}^{+}$is much higher than the one of the crustal average.

\section{Conclusions}

Aerosol sampling took place, on a 12-h time-resolution basis, in the centre of Athens, Greece, during two 20-day periods, in August 2003 and March 2004. The August 2003 campaign mean $\mathrm{PM}_{10}$ mass concentration, obtained by Beta Attenuation at $5 \mathrm{~m}$ above ground in Athinas Street, was $56 \mu \mathrm{g} \mathrm{m}^{-3}$ while the corresponding value for March 2004 was $92 \mu \mathrm{g} \mathrm{m}^{-3}$. In both campaigns the E.U. imposed daily limit of $50 \mu \mathrm{g} \mathrm{m}^{-3}$ was exceeded on several occasions. During the March campaign, in Athinas Street, additionally obtained DSFU-PM $10\left(\mathrm{PM}_{10-2.5}+\mathrm{PM}_{2.5}\right)$ gravimetric mass concentrations (mean: $121 \mu \mathrm{g} \mathrm{m}^{-3}$ ) in the "breathing zone", at $1.5 \mathrm{~m}$ above ground were significantly higher compared to the respective mean $\mathrm{PM}_{10}$ mass concentrations obtained by the same method at $25 \mathrm{~m}$ above ground, in a second site (AEDA; mean: $86 \mu \mathrm{g} \mathrm{m}^{-3}$ ) also in the centre of the city. For the fine particulate matter the Athinas St. concentrations were on average $22 \%$ higher than the respective AEDA ones, while for the coarse fraction, this discrepancy was even higher, reaching $30 \%$. The significantly higher particulate mass concentrations close to the ground were mainly due to crust-related calcium and aluminosilicate compounds and were related to a significant extend to construction as well as dust/soil re-suspension by traffic and overall human activity. $\mathrm{PM}_{10}$ mass concentration measurements near ground level, in the "breathing zone", are presented for the first time for the centre of Athens and, it is shown that, for a realistic estimation of the exposure of citizens to particulate matter in the centre of the city, knowledge of $\mathrm{PM}_{10}$ levels within the "breathing zone" is necessary.

The simultaneous sampling with a pair of DSFUs allowed for the determination of ionic and elemental concentrations.
Calcium has been found to be the predominant element in the coarse fraction, appearing in very high concentrations compared to the ones reported for other cities. Its extremely elevated near-ground concentrations have been probably connected with the extensive construction works that were taking place in the centre of the city for the Olympic Games of 2004, as well as the calcitic rocks that predominate the geology of the region. Crust-related aluminosilicates and iron have also been found to be major components of the coarse and fine fraction and sulfur has been predominant in the fine fraction. Toxic metals have been found to be well below the air quality limits and reduced compared to older studies, with the exception of $\mathrm{Cu}$ and $\mathrm{V}$ for which some increase has been recently observed. $\mathrm{Pb}$, in particular, appears mostly in the fine fraction and in very low concentrations compared to studies dating more than a decade ago.

In the coarse fraction, major ions have been found to be $\mathrm{Ca}^{2+}$ and $\mathrm{NO}_{3}^{-}$, while $\mathrm{SO}_{4}^{2-}, \mathrm{Ca}^{2+}$ and $\mathrm{NH}_{4}^{+}$were the major ionic components of the fine fraction. An important deficiency of anions has been found in the coarse fraction and is assumed to be due to the existence of great amounts of $\mathrm{CO}_{3}^{-}$, as has been verified by another recent study, and $\mathrm{OH}^{-}$; both species are not analyzed by our method. The low molar ratio of $\mathrm{NH}_{4}^{+} / \mathrm{SO}_{4}^{2-}$ has indicated an ammoniumpoor ambient air, where atmospheric ammonia is consumed with sulfate without sufficing its neutralization to full extend and the formation of $\mathrm{NH}_{4} \mathrm{NO}_{3}$ is therefore not favoured. The high correlations between $\mathrm{NH}_{4}^{+}$and $\mathrm{SO}_{4}^{2-}$ indeed suggested that in the fine particles, $\mathrm{NH}_{4}^{+}$is an important neutralizing agent of sulphuric acid forming salts like $\mathrm{NH}_{4} \mathrm{HSO}_{4}$, $\left(\mathrm{NH}_{4}\right)_{3}\left(\mathrm{SO}_{4}\right)_{2}$ and $\left(\mathrm{NH}_{4}\right)_{2} \mathrm{SO}_{4}$. In addition to this, and as expected, the respective correlations for $\mathrm{NH}_{4}^{+}$and $\mathrm{NO}_{3}^{-}$have been found low, in agreement with the prediction that formation of $\mathrm{NH}_{4} \mathrm{NO}_{3}$ does not occur to a significant extend. The relatively little fine-fraction $\mathrm{NO}_{3}^{-}$correlates considerably well with $\mathrm{Mg}^{2+}$ and $\mathrm{Ca}^{2+}$ which indicates the formation of salts like $\mathrm{Mg}\left(\mathrm{NO}_{3}\right)_{2}$ and $\mathrm{Ca}\left(\mathrm{NO}_{3}\right)_{2}$. Water-soluble ionic calcium is by far the most abundant specie in the coarse fraction. Hence, the good correlation between $\mathrm{Ca}^{2+}$ with $\mathrm{NO}_{3}^{-}$ and $\mathrm{SO}_{4}^{2-}$ suggests that calcium-containing aerosols, originating from the calcium-rich soils in the region as well as the quick-lime extensively used in painting facades in Greece, are probably the most important neutralizing species in the coarse fraction, on the surface of which acids like $\mathrm{H}_{2} \mathrm{SO}_{4}$ and $\mathrm{HNO}_{3}$ react to form $\mathrm{Ca}\left(\mathrm{NO}_{3}\right)_{2}$ and $\mathrm{CaSO}_{4}$. Strong correlations have been observed between $\mathrm{Na}^{+}$and $\mathrm{Mg}^{2+}, \mathrm{Na}^{+}$ and $\mathrm{Cl}^{-}$and $\mathrm{Mg}^{2+}$ and $\mathrm{Cl}^{-}$, which indicates the existence of marine-related $\mathrm{NaCl}$ and $\mathrm{MgCl}_{2}$.

Morning and evening means have been calculated and presented separately, as this may demonstrate trends in the "within-a-day" variation of elements and ionic species concentrations. For many days, morning concentrations were slightly higher than the evening concentrations, when most traffic and construction activity in the city was reduced. 
However, on some occasions and for some species like $\mathrm{Na}$, $\mathrm{Cl}^{-}$and $\mathrm{NO}_{3}^{-}$in the coarse fraction and $\mathrm{SO}_{4}^{2-}$ and $\mathrm{NH}_{4}^{+}$in the fine fraction, as well as a some elements, evening values were slightly higher over morning ones. This might be indicative of the trapping of near-surface aerosols under a shallow atmospheric boundary layer during the night. Total analyzed inorganic mass (elemental+ionic) has been found to range between $25-33 \%$ of the total coarse particle mass and $35-42 \%$ of the total fine particle mass. The unknown part is believed to include major ions $\left(\mathrm{CO}_{3}^{-}, \mathrm{OH}^{-}\right)$, lighter elements and other mineral compounds (with bound oxygen and nitrogen), as well as strongly bound water, carbon, organics and polymers, which usually constitute more than $50 \%$ of the urban aerosol mass.

Acknowledgements. The authors would like to thank P. Wåhlin for his analytical contribution and discussion on the PIXE analysis method. Additionally, the authors would like to thank C. Polizou for providing Beta Attenuation-obtained $\mathrm{PM}_{10}$ time-series and the Greek Ministry of Environment for providing time-series of gaseous pollutants concentrations and meteorological variables, respectively. The authors are also grateful to D. Tsoumani for her in-laboratory assistantship, and G. Andreou, E. Charpantidou and G. Loupa for their overall help during the sampling campaigns. This work has been partially funded by the European Commission under the Fifth Framework Programme, through the research project SAPPHIRE.

Edited by: R. Vautard

\section{References}

Artunano, B., Salvador, P., Alonso, D. G., Querol, X., and Alastuey, A.: Anthropogenic and natural influence on the $\mathrm{PM}_{10}$ and $\mathrm{PM}_{2.5}$ aerosol in Madrid (Spain). Analysis of high concentration episodes, Environ. Pollut., 125, 453-465, 2003.

Chaloulakou, A., Kassomenos, P., Spyrellis, N., Demokritou, P., and Koutrakis, P.: Measurements of $\mathrm{PM}_{10}$ and $\mathrm{PM}_{2.5}$ particle concentrations in Athens, Greece, Atmos. Environ., 37, 649-660, 2003.

Chaloulakou, A., Kassomenos, P., Grivas, G., and Spyrellis, N.: Particulate matter and black smoke concentration levels in central Athens, Greece, Environ. Int., 31, 651-659, 2005.

Chan, C. Y., Xu, X. D., Li, Y. S., Wong, K. H., Ding, G. A., Chan, L. Y., and Cheng, X. H.: Characteristics of vertical profiles and sources of $\mathrm{PM}_{2.5}, \mathrm{PM}_{10}$ and carbonaceous species in Bejing, Atmos. Environ., 39, 5113-5124, 2005.

Charron, A., Harrison, R. M., Moorcroft, S., Booker, J.: Quantitative interpretation of divergence between $\mathrm{PM}_{10}$ and $\mathrm{PM}_{2.5}$ mass measurement by TEOM and gravimetric (Partisol) instruments, Atmos. Environ., 38(3), 415-423, 2004.

Commission, E.: 1999/30/EC. Relating to limit values for sulphur dioxide, nitrogen dioxide and oxides of nitrogen, particulate matter and lead in ambient air, Official Journal of the European Communities, L 163/41-60, 1999.

Commission, E.: Quality of petrol and diesel fuel used for road transport in the European Union (Reporting year 2003), Re- port from the Commission, $\operatorname{COM}(2005)$, Brussels, 2.3.2005, $\operatorname{COM}(2005) 69$ final, 2005.

D' Alessandro, A., Lucarelli, F., Mando, P. A., Marcazzan, G., Nava, S., Prati, P., Valli, G., Vecchi, R., and Zucchiatti, A.: Hourly elemental composition and sources identification of fine and coarse particulate matter in four italian towns, Aerosol Sci., 34, 243-259, 2003.

Danalatos, D. and Glavas, S.: Atmospheric nitric acid concenrtations in a mediterranean site, Patras, Greece, Atmos. Environ., 29, 1849-1852, 1995.

Davis, B. L. and Jixian, G.: Airborne particulate study in five cities of China, Atmos. Environ., 34, 2703-2711, 2000.

Dockery, D. W and Pope, C. A.: Acute respiratory effects of particulate air pollution, Ann. Rev. Publ. Health, 15, 107-132, 1994.

Eleftheriadis, K., Balis, D., Ziomas, I. C., Colbeck, I., and Manalis, N.: Atmospheric aerosol and gaseous species in Athens, Greece, Atmos. Environ., 32, 2183-2191, 1998.

Fyfe, W. S.: Geochemistry, Oxford University Press, 1974.

Graham, B., Falkovich, A. H., Rudich, Y., Maenhaut, W., Guyon, P., and Andreae, M.: Local and regional contributions to the atmospheric aerosol over Tel Aviv, Israel: a case study using elemental, ionic and organic tracers, Atmos. Environ., 38, 1593-1604, 2004.

Grivas, G., Chaloulakou, A., Samara, C., and Spyrellis, N.: Spatial and temporal variation of $\mathrm{PM}_{10}$ mass concentrations within the Greater Area of Athens, Greece, Water, Air Soil Pollut., 158, 357-371, 2004.

Harrison, R. M., Smith, D. J. T., and Luhana, L.: Source Apportionment of Atmospheric Polycyclic Aromatic Hydrocarbons Collected from an urban location in Birmingham, UK, Environ. Sci. Technol., 30, 825-832, 1996.

Harrison, R.M., Jones, A., M., Lawrence, R., G.: A pragmatic mass closure model for airborne particulate matter at urban background and roadside sites, Atmos. Environ., 37, 4927-4933, 2003.

Katsouyanni, K., Touloumi, G., Spix, C., Schwartz, J., Balducci, F., Medina, S., Rossi, G., Wojtyniak, B., Sunyer, J., Bacharova, L., Schouten, P., Ponka, A., and Andersen, H. R.: Short term effects on ambient air sulphur dioxide and particulate matter on mortality in 12 European cities: results from time series data from the APHEA project, British Medical Journal, 314(7095), 1658$1663,1997$.

Katsouyanni, K., Touloumi, G., Samoli, E., Gryparis, A., Le Tertre, A., Monopolis, Y. Y., and Tossi, G.: Confounding and effect modification in the short-term effects of ambient particles on total mortality: results from 29 European cities within the APHEA2 project, Epidemiology, 12, 521-531, 2001.

Koliadima, A., Athanasopoulou, A., and Karaiskakis, G.: Particulate matter in the air of the cities of Athens and Patras (Greece): particle size distributions and elemental concentrations, Aerosol Sci. Technol., 28, 292-300, 1998.

Kouyoumdjian, H. and Saliba, N. A.: Mass concentration and ion composition of coarse and fine particles in an urban area in Beirut:effect of calcium carbonate on the absorption of nitric and sulfuric acids and the depletion of chloride, Atmos. Chem. Phys., 6, 1865-1877, 2006, http://www.atmos-chem-phys.net/6/1865/2006/.

Luhana L., H. Mao, and Sokhi, R. S.: Laboratory and field evaluation of UH Dichotomous Stacked Filter Units (DSFU), Univer- 
sity of Hertfordshire, SAPPHIRE Report (EVK4-2001-00192), 2001.

Manalis, N., Grivas, G., Protonotarios, V., Moutsatsou, A., Samara, C., and Chaloulakou, A.: Toxic metal content of particulate matter $\left(\mathrm{PM}_{10}\right)$, within the Greater Area of Athens, Chemosphere, 60, 557-566, 2005.

Marcazzan, G. M., Vaccaro, S., Valli, G., and Vecchi, R.: Characterisation of $\mathrm{PM}_{10}$ and $\mathrm{PM}_{2.5}$ particulate matter in the ambient air of Milan (Italy), Atmos. Environ., 35, 4639-4650, 2001.

Miller, J. C. and Miller, J. N.: Statistics for Analytical Chemistry, Ellis Horwood PTR Prentice Hall, England, 1993.

Pakkanen, T. A., Loukkola, K., Korhonen, C. H., Aurela, M., Mäkelä, T., Hillamo, R. E., Aarnio, P., Koskentalo, T., Kousa, A., and Maenhaut, W.: Sources and chemical composition of atmospheric fine and coarse particles in the Helsinki area, Atmos. Environ., 35, 5381-5391, 2001.

Pathak, R. K., Louie, P. K. K., and Chan, C. K.: Characteristics of aerosol acidity in Hong Kong, Atmos. Environ., 38, 2965-2974, 2004.

Pathak, R. K. and Chan, C. K.: Inter-particle and gas-particle interactions in sampling artifacts of $\mathrm{PM}_{2.5}$ in filter-based samplers, Atmos. Environ., 39, 1597-1607, 2005.

Peng, R. D., Dominici, F., Pastor-Barriuso, R., Zeger, S. L., and Samet, J. M.: Seasonal analyses of air pollution and mortality in 100 US, Am. J. Epidemiol., 161, 585-594, 2005.

Pope III, C. A., Burnett, R. T., Thun, M. J., Cale, E. E., Krewski, D., Ito, K., and Thurston, G. D.: Lung cancer, cartiopulmonary mortality and long-term exposure to fine particulate air pollution, J. Am. Medical Assoc., 287, 1132-1141, 2002.

Puxbaum, H., Gomiscek, B., Kalina, M., Bauer, H., Salam, A., Stopper, S., Preining, O., and Hauck, H.: A dual site study of $\mathrm{PM}_{2.5}$ and $\mathrm{PM}_{10}$ aerosol chemistry in the larger region of $\mathrm{Vi}$ enna, Austria, Atmos. Environ., 38, 3949-3958, 2004.

Querol, X., Alastuey, A., Rodriguez, S., Plana, F., Ruiz, C. R., Cots, N., Massague, G., and Puig, O.: $\mathrm{PM}_{10}$ and $\mathrm{PM}_{2.5}$ source apportionment in the Barcelona Metropolitan area, Catalonia, Spain, Atmos. Environ., 35, 6407-6419, 2001.

Salma, I., Maenhaut, W., Zemplen-Papp, E., and Zaray, G.: Comprehensive characterization of atmospheric aerosols in Budapest, Hungary: physicochemical properties of inorganic species, Atmos. Environ., 35, 4367-4378, 2001.

Scheff, P. A. and Valiozis, C.: Characterization and source identification of respirable particulate matter in Athens, Atmos. Environ., 24A, 203-211, 1990.

Seinfeld, J. H. and Pandis, S. N.: Atmospheric Chemistry and Physics: From air pollution to climate change, John Wiley \& Sons, 1998.

Shaka, H. and Saliba, N. A.: Concentration measurements and chemical composition of $\mathrm{PM}_{10-2.5}$ and $\mathrm{PM}_{2.5}$ at a coastal site in Beirut, Lebanon, Atmos. Environ., 38, 523-531, 2004.
Sillanpää, M., Hillamo, R., Saarikoski, S., Frey, A., Pennanen, A., Makkonen, U., Spolnik, Z., Van Grieken, R., Branis, M., Brunekreef, B., Chalbot, M., Kuhlbusch, T., Sunyer, J., Kerminen, V., Kulmalak, M., Salonen, R. O.: Organic, elemental and inorganic carbon in particulate matter of six urban environments in Europe, Atmos. Chem. Phys., 5, 2869-2879, 2005, http://www.atmos-chem-phys.net/5/2869/2005/.

Sillanpää, M., Frey, A., Hillamo, R., Pennanen, A. S., and Salonen, R. O.: Chemical composition and mass closure of particulatematter at six urban sites in Europe, Atmos. Environ., 40, S212S223, 2006.

Siskos, P., Bakeas, E., Lioli, I., Smirnioudi, V. N., and Koutrakis, P.: Chemical characterization of $\mathrm{PM}_{2.5}$ aerosols in Athens - Greece, Environ. Technol., 22, 687-695, 2001.

Thomaidis, N. S., Bakeas, E., and Siskos, P.: Characterization of lead, cadmium, arsenic and nickel in $\mathrm{PM}_{2.5}$ particles in the Athens atmosphere, Greece, Chemosphere, 52, 959-966, 2003.

Symmeonidis, P.: Study of atmospheric pollution and its sources by use of GIS, PhD Thesis (in Greek), Aristotle University of Thessaloniki, 2002.

Touloumi, G., Pocock, S. J., Katsouyanni, K., and Trichopoulos, D.: Short term effects of air pollution on daily mortality in Athens. A time series analysis, Int. J. Epidemiol., 23, 957-967, 1994.

Trebs, I., Metzger, S., Meixner, F. X., Helas, G., Hoffer, A., Rudich, Y., Falkovich, A. H., Moura, M. A. L., Da Silva Jr., R. S., Artaxo, P., Slanina, J., and Andreae, M.: The $\mathrm{NH}_{4}^{+}-\mathrm{NO}_{3}^{-}-\mathrm{Cl}^{-}-\mathrm{SO}_{4}^{-2}-$ $\mathrm{H}_{2} \mathrm{O}$ aerosol system and its gas phase precursors at a pasture site in the Amazon Basin: How relevant are mineral cations and soluble organic acids?, J. Geophys. Res., 110, 1-18, 2005.

Tsitouridou, R. and Samara, C.: First results of acidic and alkaline constituents determination in air particulates of Thessaloniki, Greece, Atmos. Environ., 27B, 313-319, 1993.

Valavanidis, A., Fiotakis, K., Vlahogianni, T., Bakeas, E. B., Triantafyllaki, S., Paraskevopoulou, V., and Dassenakis, M.: Characterization of atmospheric particulates, particle-bound transition metals and polycyclic aromatic hydrocarbons of urban air in the centre of Athens (Greece), Chemosphere, 2006.

WHO: Air Quality Guidelines, 2nd ed., WHO Regional Office for Europe, Copenhagen, Denmark, 2000.

Xie, R. K., Seip, H. M., Leinum, J. R., Winje, T., and Xiao, J. S.: Chemical characterization of individual particles $\left(\mathrm{PM}_{10}\right)$ from ambient air in Guiyang City, China, Sci. Total Environ., 343, 261-272, 2005.

Yaaqub, R. R., Davis, T. D., Jickells, T. D., and Miller, J. M.: Trace elements in daily collected aerosols at a site in southeast England, Atmos. Environ., 25A, 985-996, 1991.

Yatin, M., Tuncel, S., Aras, N. K., Olmez, I., Aygun, S., and Tuncel, G.: Atmospheric trace elements in Ankara, Turkey: 1. factors affecting chemical composition of fine particles, Atmos. Environ., 34, 1305-1318, 2000. 